\title{
Multi-level and Multi-Objective Design Optimisation of a MEMS Bandpass Filter
}

\author{
Michael Farnsworth ${ }^{\mathrm{a}, *}$, Ashutosh Tiwari ${ }^{\mathrm{a}}$, Meiling Zhu ${ }^{\mathrm{b}}$ \\ ${ }^{a}$ School of Aerospace, Transport Systems and Manufacturing, Cranfield University, College Road, \\ Bedfordshire, MK43 OAL, UK \\ ${ }^{b}$ College of Engineering, Mathematics and Physical Systems, University of Exeter, EX4 4 SB
}

\begin{abstract}
Microelectromechanical system (MEMS) design is often complex, containing multiple disciplines but also conflicting objectives. Designers are often faced with the problem of balancing what objectives to focus upon and how to incorporate modeling and simulation tools across multiple levels of abstraction in the design optimization process. In particular due to the computational expense of some of these simulation methods there are restrictions on how much optimization can occur. In this paper we aim to demonstrate the application of multi-objective and multi-level design optimisation strategies to a MEMS bandpass filter. This provides for designers the ability to evolve solutions that can match multiple objectives. In order to address the problem of a computationally expensive design process a novel multi-level evaluation strategy is developed. In addition a new approach for bandpass filter modeling and optimization is presented based up the electrical equivalent circuit method. In order to demonstrate this approach a comparison is made to previous attempts to design similar bandpass filters. Results are comparable in design but at a significant reduction in functional evaluations, needing only 10,000 functional evaluations in comparison to 2.6 million with the previous work.
\end{abstract}

Keywords: MicroElectroMechanical Systems, MEMS, MOEA, Multi-Objective Optimisation, Multi-level Optimisation

\section{Introduction}

Automation of design is an important part of any successful approach to optimize or develop new products. There are a number of ways in which a designer can be aided in improving this efficiency for example the use of state of the art modeling and simulation tools, to automated forms of design synthesis and optimization. One field of manufacturing to benefit from the use of modeling, simulation and soft computing tools is that found in the design and manufacture of microelectromechanical systems (MEMS). The multidisciplinary and hierarchical nature of MEMS design makes balancing the process between any number of simulation and

\footnotetext{
*Corresponding author

Email address:

m.j.farnsworth@cranfield.ac.uk (Meiling Zhu)
}

Preprint submitted to Applied Soft Computing modeling tools to achieve the desired outcome and speeding up this process difficult. This is in conjunction with the often large number of conflicting objectives and constraints that designers have to take into account when creating new applications or designs. Strategies that utilize and exploit the large number of modeling and simulation tools available could prove a solution to overcome the barrier of using computationally expensive modeling simulation tools, while multi-objective optimisation algorithms can allow for solutions that meet or balance the needs of the designer.

Past work on design optimization of MEMS focuses upon a single level strategy, where a single optimization or meta-heuristic algorithm is applied in an iterative fashion $\left[{ }^{9}\right]\left[{ }^{15}\right]\left[{ }^{43}\right]$. However the nature of MEMS design synthesis and optimization brings with it many challenges that such an approach will struggle with. An example is the cost of model-

October 4, 2016

Published by Elsevier. This is the Author Accepted Manuscript issued with: 
ing and simulation for fitness evaluation that can reduce the number of solutions a designer is able to create and evaluate. A novel solution to this is in the application of a multi-level strategy that exploits different levels of modeling and simulation abstraction to lower evaluation cost, speed up design and allow for increased search of the design space. In tandem a novel electrical circuit equivalent model is developed to provide different levels of abstraction and functional evaluation cost to enable the application of a multi-level strategy for MEMS bandpass filter design.

\section{Related Research}

\subsection{Microelectromechanical systems}

Microelectromechanical systems are a multidisciplinary field built from the Very-Large-ScaleIntegration (VLSI) fabrication techniques originally the foundation of the integrated circuit (IC) community. Their application covers a wide range of disciplines from the commercial, industrial and academic domains, including not just the mechanical and electrical $\left[^{1}\right]$ but also the fluidic $\left[^{2}\right]$, thermal $\left[{ }^{3}\right]$, chemical, biological $\left[^{4}\right]$ and magnetic $\left[{ }^{5}\right]$ phenomena. These include but are not limited to gyroscopes $\left[{ }^{6}\right]$, micro-resonators $\left[{ }^{7}\right]$, and energy harvesters $\left.{ }^{8}\right]$. Tied to these areas are a host of modeling and simulation tools, often based around the hierarchical nature of the MEMS design process. Here the design process is often decomposed into four levels (System, Device, Physical, and Process) with a focus upon varying levels of abstraction and system to component view. The system level focuses upon the use of lumped element circuit models or block diagrams to model device performance, utilising powerful circuit simulators. They provide the possibility to interface with the mechanical elements of the device, either through analytical models, HDL models, reduced order models or alternatively electrical equivalent representations of the mechanical component. Both the device and physical level provide models of varying granularity. At a device level, a designer can look to build accurate 2D layout models through the use of NODAL simulators and various atomic MEMS elements, or by building mathematical analytical representations. The two major contributions to nodal analysis and its application to MEMS design are the tools NODAS [ $\left.{ }^{44} 45\right]$ and Sugar $\left[{ }^{12}\right]$. The development of NODAS derived from the Nodal De- sign of Actuators and Sensors in $\left.{ }^{45}\right]$ was motivated by the need to overcome constraints of previous behavioural modelling which focused on individual devices as a whole. The physical level generally utilises more expensive finite element and boundary element methods to simulate and analyse 3D models of the device. The process level looks towards the creation of appropriate mask layouts and process information needed for the batch process generally employed to fabricate the device. Therefore, by utilising system level tools it is possible to derive the function of the whole coupled electromechanical device, while the device or physical levels allow the device to be envisioned and thus allow fabrication to follow function. Traditionally the design of MEMS devices has followed a tried and tested hand-driven or back of the envelope approach with the addition of modeling and simulation tools to allow for visualization and analysis of the physical behaviour of the device. However in recent years there has been a drive to automate the design and development of MEMS devices by removing the dependence on hand-driven design and optimization and looking to develop automated algorithms and heuristics tailored towards MEMS design synthesis and optimization.

\subsection{Conventional vs Unconventional De- sign Synthesis}

Traditional design synthesis and optimisation by MEMS designers has often followed a trial-anderror approach with new designs being fabricated, tested to failure and then if necessary followed up with further redesign. This is both time consuming and expensive. The advent of modeling and simulation tools has enabled designers to perform this process in-silica but it is still very much dependent on a particular designers skill set, and with MEMS being highly multidisciplinary this can have its disadvantages. The introduction of automation into the process to improve design synthesis and optimisation is an approach which has taken hold over the past decades. To begin with this often focused around simple gradient based methods $\left[{ }^{9}\right]\left[{ }^{10}\right]$ looking to solve simple single objectives to more complex stochastic algorithms such as those found in the field of evolutionary computation. Early work done by Zhou $\left.{ }^{11}\right]$ looked to apply multi-objective genetic algorithms to the design and optimisation of a number of device level MEMS devices using a NODAL simulator $\left[{ }^{12}\right]$ and over the years the use 
of evolutionary computational methods in MEMS design has expanded into a number of areas such as conceptual design [ $\left.{ }^{13}\right]$, component based design, classical shape $\left.{ }^{14}\right]$, sizing $\left[^{15}\right]$ and topological [16] design optimisation to interactive $\left.{ }^{17}\right]$ and casebased reasoning methods $\left.{ }^{18}\right]$. A comprehensive list of conventional and unconventional methods for MEMS design optimisation can be found in [19]. Hierarchical or multi-level methods are another unconventional approach which looks to utilize their specific architecture to benefit the design optimisation process. This is often in the form of either hybridizing multiple methods of search for example local gradient based methods with more exploratory stochastic methods to improve the search capabilities $\left[{ }^{20}\right]\left[{ }^{21}\right]$, multiple approaches to parameterization of the variables or constraints present in the model to be optimized $\left[{ }^{22}\right]\left[{ }^{23}\right]$, or multiple methods of evaluation for different abstractions or modeling disciplines $\left[{ }^{23}\right]\left[{ }^{24}\right]\left[{ }^{25}\right]\left[{ }^{26}\right]\left[{ }^{27}\right]$.

\section{MEMS Bandpass Filter Modelling}

Microelectromechanical systems offer a range of benefits over their contemporary macro alternatives and more and more are giving rise to replacing them. Often more robust and environmentally tolerant $\left[{ }^{28}\right]$, they also benefit from their scale by taking up less space and requiring less power to function. Heterodyning communications are one area where the application of MEMS could provide profound benefits $\left[{ }^{29}\right]$. A component of such communication devices are bandpass filters, particularly important in highly selective HF (high-frequency), IF (intermediate-frequency), or RF (radio-frequency) signal processing and mixing [30]. The performance characteristics of such filters such as having a low insertion loss, small percent bandwidth and shape factor are heavily influenced by the Quality (Q) factor of the component which itself must be tolerant also to environmental variations such as temperature, noise or microphonics that effect frequency transmission $\left[{ }^{29}\right]\left[{ }^{31}\right]$. Electronic filters, such as transistor based LC circuits can be employed to function as filtering components; however they are limited due to their relatively low $\mathrm{Q}$ factor values $\left.{ }^{32}\right]$. The majority of heterodyne transceivers utilize macro vibrating mechanical tank components like crystal or surface acoustic wave (SAW) resonators $\left[{ }^{31}\right]\left[{ }^{33}\right]$ to perform signal processing. These particular components have advantages over tran- sistor based technologies in comparable filters due in part to their high-Q factor, giving rise to superior performance in insertion loss, percentage bandwidth, and achievable rejection, and also their stability against thermal ageing $\left[{ }^{31}\right]\left[{ }^{34}\right]\left[{ }^{35}\right]$. The mechanical components outlined however do have a particular disadvantage in that they are off-chip and therefore must interface with processing circuitry at the board level, increasing total device area. This is problematic with regards to miniaturization of the system and therefore portability of wireless transceivers that has led to research on new strategies for miniaturization of these components $\left[{ }^{31}\right]\left[{ }^{36}\right]\left[{ }^{29}\right]$. CMOS micromachining technology allows for the fabrication of on-chip components that can interface directly with electronic interface circuitry and therefore reduce the overall device footprint. This technology has been employed in the synthesis of High-Q micro mechanical oscillators/resonators [ $\left.{ }^{33}\right]$ that match target performance and cost goals designers aim for. Laterally driven folded beam micromechanical resonators offer on-chip alternative devices that provide high-Q filtering, reduce size and energy consumption, and are integrated with electrical components directly $\left[{ }^{33}\right]\left[{ }^{31}\right]$. The device itself consists of a suspended interdigited mass held up by an anchored folded flexure spring component, where the device as a whole is driven and sensed via an electrostatic capacitive comb transducer. An extensive overview on MEMS resonator and oscillator devices is covered in [ $\left.{ }^{30}\right]$. The coupling of laterally driven microresonators can be used to create bandpass filters, where the resonator is driven by an interdigitated comb transducer while another acts as an electrostatic sensing receiver $\left[{ }^{30}\right]$. A capacitive comb transducer utilises both the mechanical and electrical energies of a system in an equilibrium where an input electrical signal in the form of current or voltage is converted into a mechanical displacement $\left[{ }^{33}\right]\left[{ }^{31}\right]\left[{ }^{37}\right]$. These mechanical vibrations are processed through the mechanical domain of the filter and then converted back into electrical signals by an output sensing comb transducer [ $\left.{ }^{37}\right]$. The expression $\partial C_{n} / \partial x$ can therefore be approximated as:

$$
\frac{\partial C_{n}}{\partial x}=\frac{2 \xi N_{f i n} \epsilon_{0} h}{d}
$$

Where $\xi$ is a constant that models additional capacitance due to fringing electric fields, $N_{\text {fin }}$ is the number of finger gaps within the comb drive, 
$\epsilon_{0}$ is the permittivity of air, $h$ is the structural layer thickness and $d$ is the comb finger gap spacing $\left.{ }^{38}\right]\left[{ }^{33}\right]$. It is the output signal which forms the characteristic frequency transmission required for filter signal processing. The central frequency of an individual resonator and filter as a whole is dependent on the resonance frequency of the individuals resonators, the bandwidth characteristics of the filter are dependent upon the coupling springs and its physical properties, in particular its stiffness in the driving direction $\left[{ }^{30}\right]$.

A single folded flexure resonator exhibits a biquad frequency response required for a high-Q bandpass filter as shown in figure 1a. In a network topology of multiple resonators, each resonator exhibits its own unique biquad frequency response as shown in figure $1 \mathrm{~b}$ and adds it to the coupled system as a whole. The spacing and shape of the filter is affected by a number of variables, in particular coupling spring stiffness, the variation of which can alter the shape to give a desirable flat pass band for a filter of this type as shown in figure 1c, additional electronics and techniques such as resistance or Q-adjustor components can also be utilized for transmission shape control.

Some common characteristics of a bandpass filter are shown in figure 2 highlighting important components of a successful filter, such as bandwidth, insertion loss, stop band attenuation, and shape factor $\left.{ }^{33}\right]$. This particular filter shape can be readily achieved through the coupling of two or more resonator tanks giving rise to a coupled bandpass biquad network topology. The coupling spring of the mechanical network topology looks to effectively pull the resonator frequencies apart, creating two closely space resonator peaks that constitute the end of the filter pass band [ $\left.{ }^{31}\right]$. The number of resonators within the coupled filter network constitutes the order of a particular bandpass filter. The order plays an important role in the overall filter characteristics with higher order filters giving a sharper roll off and smaller shape factor leading to higher selectivity, however at a cost of increased insertion loss $\left[{ }^{33}\right]$. An example of a $3 r d$ order filter can be found in $\left.{ }^{33}\right]$.

The modelling of a general N-resonator series filter can be accomplished mechanically using a mechanical equivalent circuit of a single resonator device, modelled as a spring mass damper system and then through coupling of multiple resonators through the use of a soft mechanical spring [ $\left.{ }^{37}\right]$.
Table 1: Mechanical to electrical equivalence in the force-current analogy

\begin{tabular}{cc}
\hline Mechanical Variable & Electrical Variable \\
\hline Damping, $c^{-1}, k^{-1}$ & Resistance, $R$ \\
Stiffness $^{-1}$ Capacitance, $C$ \\
Mass, $m$ & Inductance, $L$ \\
Force, $f$ & Voltage, $V$ \\
Velocity, $v$ & Current, $l$ \\
Momentum, $p$ & Magnetic flux linkage, $\psi$ \\
Displacement, $x$ & Charge, $q$ \\
\hline
\end{tabular}

In figure 3a a lumped parameter mechanical circuit model of a resonator consists of the components for mechanical mass $\left(m_{r}\right)$, stiffness $\left(k_{r}\right)$ and damping $\left(c_{r}\right)$ and through coupling of a weak spring $\left(k_{s}\right)$ can be configured into a 2nd order bandpass filter as seen in figure $3 \mathrm{~b}$. A common approach towards analysis of filter designs is to transform the mechanical elements into their equivalent electrical elements using the analogy modelling method $\left[{ }^{33}\right]\left[{ }^{31}\right]\left[{ }^{37}\right]$. Table 1 outlines a number of indirect analogies between mechanical and electrical variables.

The analogous link between the two disciplines means that the force $(F)$ and velocity $(v)$ of a mechanical system can be treated as current $(I)$ and voltage $(V)$ in an electrical system. The development of equivalent circuit representations is based on the analogy in the mathematical descriptions that exists between electric and mechanical phenomena $\left[{ }^{39}\right]$. Similarities in the equations governing the behaviour of electric and mechanical systems are where the analogies are drawn and this is illustrated in Newtons second law of motion. Here the relation of force $F$ and velocity $u$ for a rigid mass $m$, is arranged as:

$$
F=m \frac{d u}{d t}=m \frac{d x^{2}}{d t^{2}}
$$

and the subsequent electric equivalent an inductor relates as:

$$
v=L \frac{d i}{d t}=L \frac{d q^{2}}{d t^{2}}
$$

In this analogy the force $F$ plays the same role as the voltage $v$, the velocity $u$ as the current $i$, and the displacement $x$ as charge $q\left[{ }^{39}\right]$. A series LCR circuit is equivalent to a 1 DOF mechanical mass-spring-damper system. Bandpass filters can 


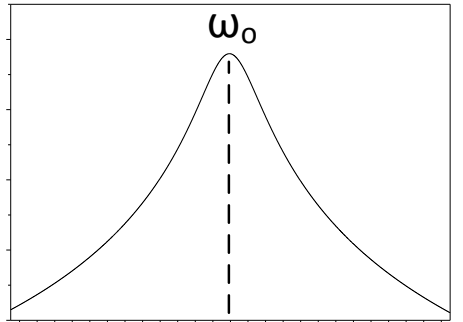

(a)

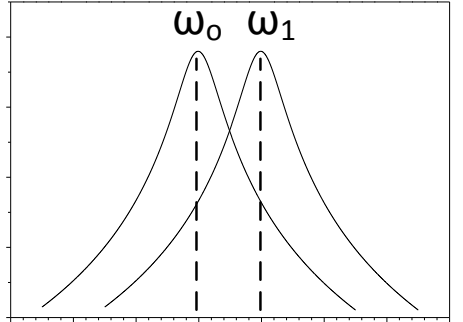

(b)

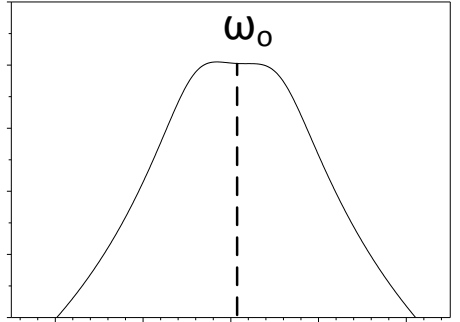

(C)

Figure 1: Filter frequency characteristics for a (a) single resonator, (b) two separate resonators and (c) a coupled two resonator system

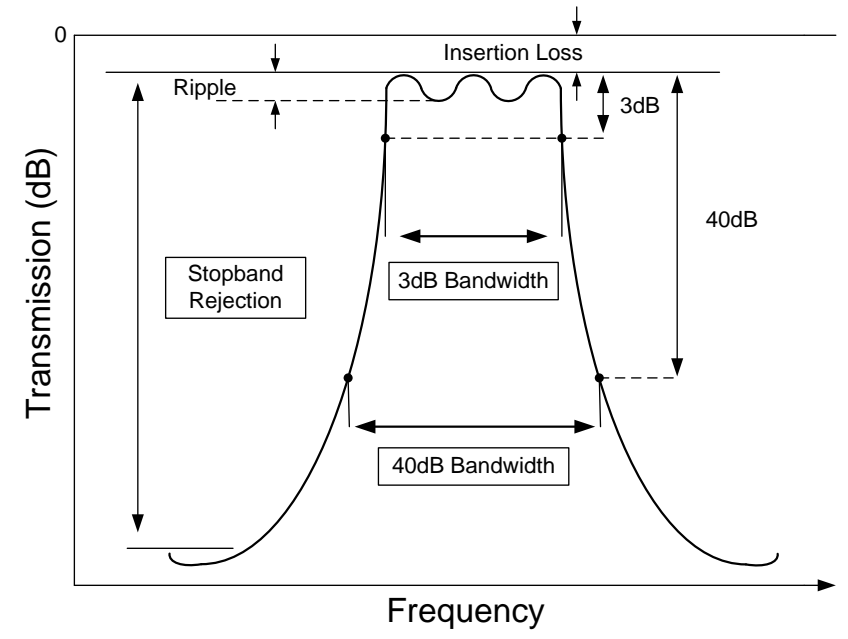

Figure 2: Bandpass filter parameter specifications $[33]$ be designed through the use of electromechanical analogies, where the electrical domain inductance, capacitance and resistance of a LCR ladder filter can be implemented via analogous values of mass, stiffness, and damping in the mechanical domain $\left.{ }^{38}\right]$. The folded flexure resonators outlined previously can be equated to LCR tanks within the electrical domain, and when coupled together using coupling shunt capacitors form a filter network $\left[{ }^{38}\right]\left[{ }^{30}\right]$.

Looking into an electrical port, with all other ports grounded of the electrical equivalent circuit of figure 4, a transformed LCR circuit is seen, with element values directly or inversely proportional to the mechanical circuit element values at the shuttle location modified or transformed by the electromechanical coupling parameter $\eta_{e n}\left[{ }^{31}\right]\left[{ }^{33}\right]$. The approach of relating the electrical equivalent element values with the mechanical equivalent values through transformation, allows the formulation of the electrical equivalent circuit using actual values for mass, stiffness and damping as the values for the inductance, capacitance, and resistance in an LCR circuit $\left[{ }^{33}\right]\left[{ }^{38}\right]$.

$$
\begin{aligned}
& R_{x n}=\frac{c_{r s}}{\eta_{e n}^{2}}=\frac{\sqrt{k_{r s} m_{r s}}}{Q \eta_{e n}^{2}} \\
& L_{x n}=\frac{m_{r s}}{\eta_{e n}^{2}} \\
& C_{x n}=\frac{\eta_{e n}^{2}}{k_{r s}} \\
& \eta_{e n}=V_{p n} \frac{\vartheta C_{n}}{\vartheta x}
\end{aligned}
$$




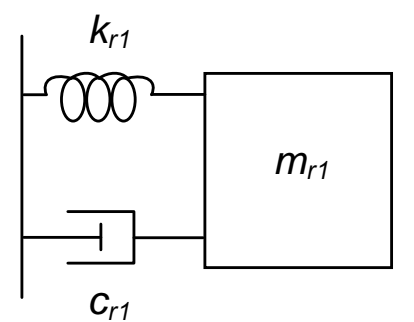

(a)

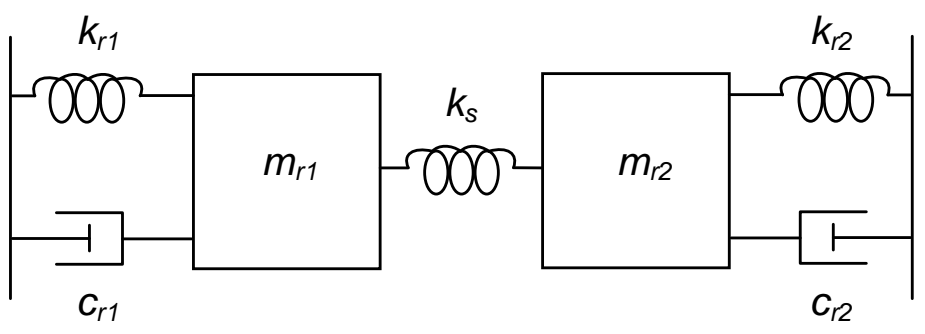

(b)

Figure 3: Lumped mechanical equivalent of a micro resonator device (a) and a 2nd order bandpass filter (b)

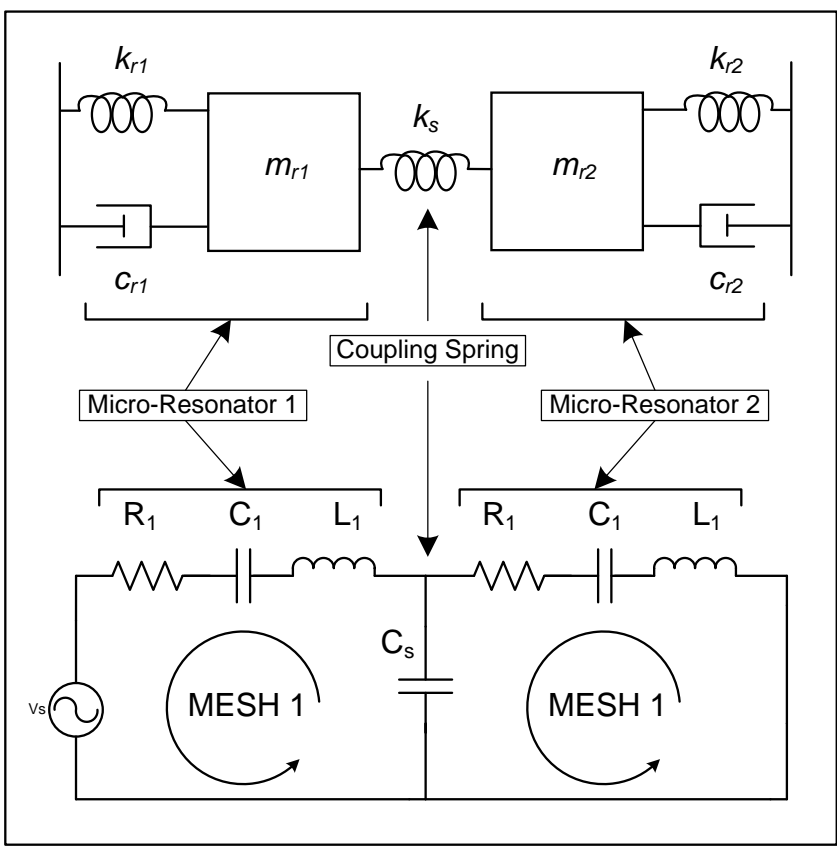

Figure 4: (Top) Lumped mechanical equivalent model of a 2nd order bandpass filter with massless coupling springs, (Bottom) corresponding electrical equivalent LCR network neglecting static capacitance at the I/O ports and utilizing an I-type coupling shunt capacitor

$$
\frac{\vartheta C_{n}}{\vartheta x}=\frac{2 \xi N_{f i n} \epsilon_{0} h}{d}
$$

The equations relating the mechanical and electrical values to each other are shown above, equations 4 - 8. The electromechanical transformation coupling parameter $\eta_{e n}$ allows for the synthesis of an LCR filter to be undertaken entirely within the electrical domain and later converting to equivalent mechanical values for device level synthesis later $\left[{ }^{31}\right]$

\section{Methodology}

The improved design synthesis and optimization of MEMS devices is the targeted outcome of this approach through the application of automated optimization heuristics in conjunction with available MEMS modeling and simulation tools. From the field of evolutionary computation two of the most popular multi-objective algorithms have been chosen to undertake design synthesis, firstly NSGAII $\left.{ }^{40}\right]$ and finally SPEA2 $\left[{ }^{41}\right]$. Both algorithms have been explored in terms of performance and applied successfully over a number of areas and problems outside and within MEMS design synthesis. The design problem revolves around the optimization of a MEMS bandpass filter. Outlined in the previous section the modeling of a bandpass filter can be achieved through the use of electrical equivalent representations. Here a design and optimisation approach looks to couple a multi-objective evolutionary algorithm with an electric circuit model representation coined (GAECM).

Bandpass filter transmission represents the main qualifier for performance of the designed LCR bandpass network; the components of such can be broken down into a set of simple characteristics similar to those in figure 2. Characterized in figure 2 is an idealized frequency transmission for a band pass filter, where the shape consists of two distinct regions, the pass band and the stop band. In an ideal bandpass filter the pass band, the targeted frequency range where signals are unfiltered, is signified by a completely flat transmission with zero insertion loss and no gain. Outside of the targeted pass band lies the stop band regions, here all incoming signals are attenuated away, in essence filtered, depending on 


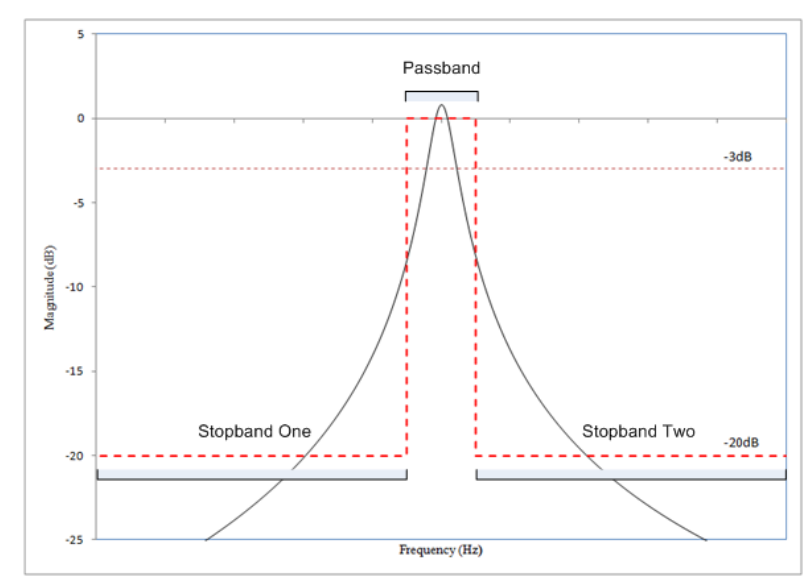

Figure 5: MEMS bandpass filter synthesis breakdown for filter objective

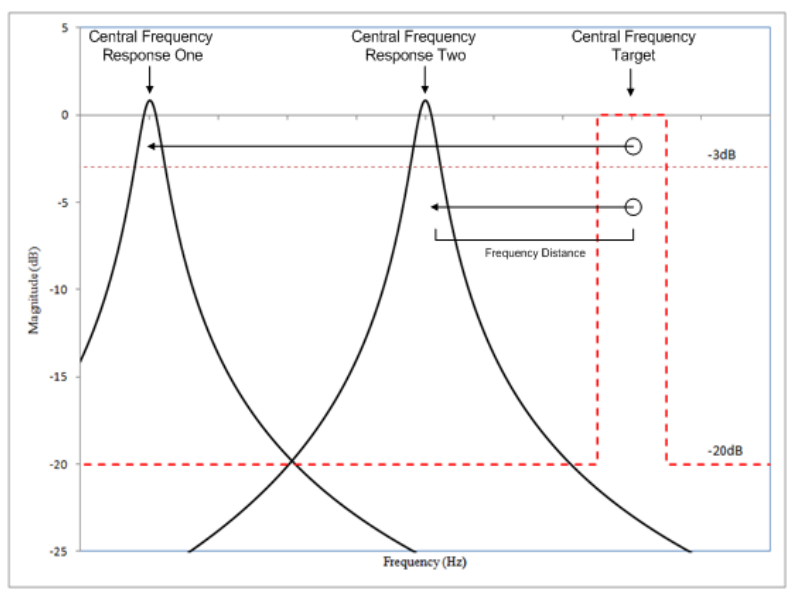

Figure 6: MEMS bandpass filter synthesis breakdown for frequency objective

the application this can lay between 20 and $120 \mathrm{~dB}$ of the nominal pass band attenuation. Ideally the drop from pass band to stop band is instantaneous.

In reality no filter is ideal, due to the attenuation just outside the pass band not providing complete rejection of the signal, this roll off is often designed to be as narrow as possible. However this representation does provide a reasonable template of characteristics in which to evaluate and quantify new filter designs against targets for pass band and stop band frequency ranges. Therefore two design objectives can be constructed as a means to evaluate new designs created by the optimizer chosen to undertake the design optimisation of the MEMS bandpass filter and are outlined in figures
5 and 6 . A frequency transmission from a single micromechanical resonator similar to that shown in figure 5 consists of a number of frequency data points plotted against the magnitude in units of $\mathrm{dB}$. The quality and performance of the filter transmission can be measured by simply calculating where each data point lies within the pass band and stop band ranges outlined and measured against their target magnitude, in this case $0 \mathrm{~dB}$ for points within the passband and $-20 \mathrm{~dB}$ within the stop band regions. The overall frequency performance can then be quantified as a sum of the total deviation from each of these ranges for the data points within the frequency transmission. Ideally all data points that lie within the pass band will have 0 insertion loss and no gain giving a magnitude of $0 \mathrm{~dB}$, while all points within the stop band will be $-20 \mathrm{~dB}$ and therefore have a deviation of 0 for both regions. Central frequency of the bandpass filter is important when wanting to design a frequency transmission for a targeted portion of the spectrum. The central frequency of a transmission is simply calculated as the distance of the peak frequency data point to the desired central frequency outlined by the designer. The objective shown in figure 6 is both a targeted design goal and a guide to the optimizer, allowing individual or coupled resonator transmission responses to move closer to the targeted region of interest. As outlined in figure 4 the components of interest are the individual LCR tanks, the coupling capacitor shunts and the values that make up equations 7 and 8 which are directly related to the comb transducer element of the filter. The individual values and the overall network topology of the circuit model produce the frequency transmission that is then used to evaluate the solution. In order to effectively simulate and analyse the circuit model solutions produced a circuit simulator is needed for the required $\mathrm{AC}$ analysis. SPICE $\left.{ }^{42}\right]$ is a common analog electronic circuit simulator that allows the constructed electrical equivalent bandpass filter circuit models to be analysed for their frequency transmission, and it has already been utilised in similar filter design synthesis $\left[{ }^{33}\right]\left[{ }^{32}\right]$. A table of the variables and objectives is shown in table 2. A bandpass filter analysis module acts as a link between the design optimisation framework used in this research and the simulator software used to analyse the individual solutions. The module constructs a parameter object using the values present in the solution and then uses this to override a bandpass fil- 
Table 2: Bandpass Filter Problem Information

\begin{tabular}{cccc}
\hline Variable Tag & Sub Tree Type & Lower Bound & Upper Bound \\
\hline Voltage & Real Valued & 1 & 200 \\
Tank Number & Integer & 1 & 9 \\
Finger Number & Integer & 1 & 200 \\
Thickness (m) & Real Valued & 2 & 30 \\
Capacitance (F) & Real Valued & $1 \mathrm{E}-15$ & $1 \mathrm{E}-11$ \\
Inductance (H) & Real Valued & 10 & 100000 \\
Tpring Capacitance (F) & Real Valued & $1 \mathrm{E}-15$ & $1 \mathrm{E}-11$ \\
Tank & Branch & $\mathrm{N} / \mathrm{A}$ & \\
\hline Objectives & & Constraints & \\
\hline Bandpass Filter Response Error & Minimize & N/A & \\
Bandpass Central Frequency Error & Minimize & & \\
\hline
\end{tabular}

ter netlist file used by the SPICE simulator for analysis. Module specific parameters allow for analysis to occur over any specific range and any number of sampling points and after analysis output data is retrieved for evaluation. The multi-objective genetic algorithms NSGAII and SPEA2 are chosen as the optimizer tasked with evolving and optimising solutions to the bandpass filter problem.

Structural tags relate to specific branch nodes within the representation and the nodes that control the count or number present. In this particular problem representation, the tank number variable and subsequently its value, has control over the tank branch node and increasing or decreasing this value leads to the addition or removal of the specific tank subtree structure. This effect is most evident within the polynomial mutation module within both NSGAII and SPEA2, where changing the value of a structure controlling variable such as tank number can lead to effectively cloning or removal of a randomly chosen structure as highlighted in figures 8 and 9.

Each node within the representation can contain a set of markers which can be used as some form of identifier or data object. These node markers can act as useful pieces of information or tools which can then be exploited to provide a diverse range of actions depending on the actions of modules that might utilise them. A simple example is shown in figure 7. The standard SBX operator within NSGAII and SPEA2 has been modified to allow control over what nodes to perform the crossover operation or not, dependent on whether the nodes themselves are marked to do so. Therefore all nodes that have a marker for SBX Crossover have that particular operation performed upon them, while those that dont are ignored. In the bandpass fil-

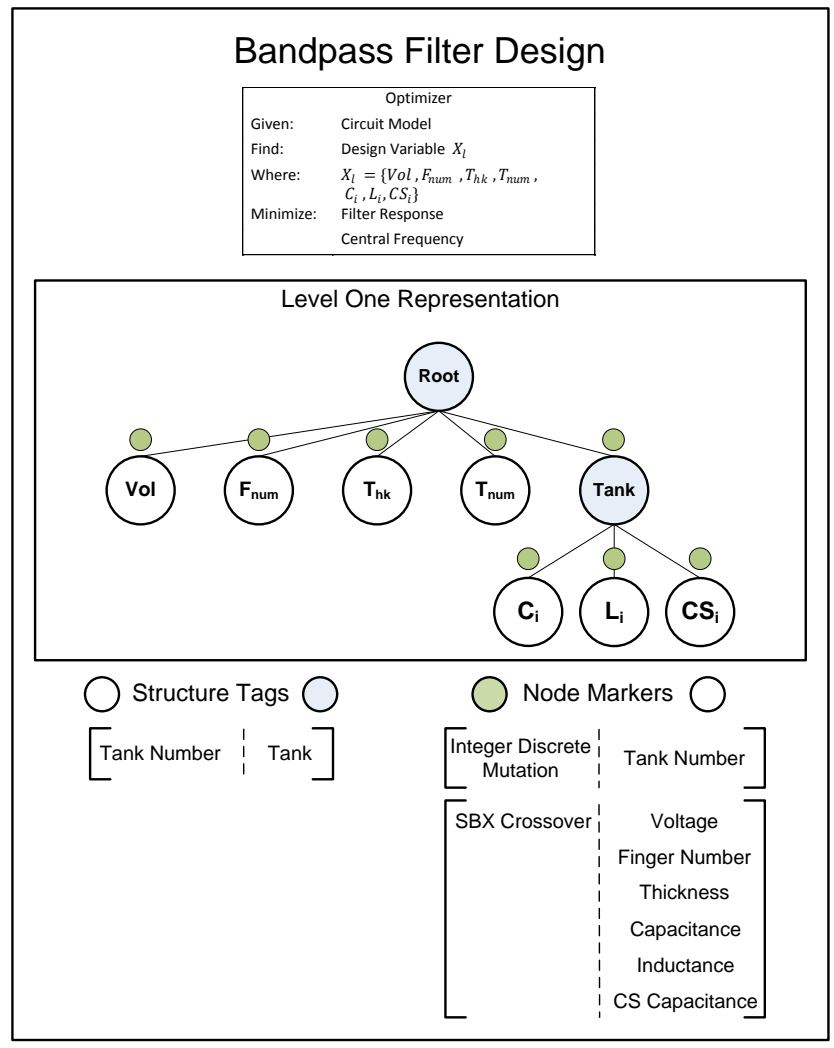

Figure 7: Bandpass Filter design template, with overview of problem, default representation, associated structure tags and node markers 


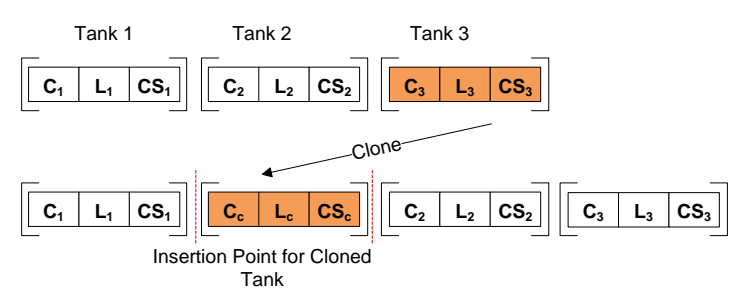

Figure 8: Structural cloning of tank component within problem representation

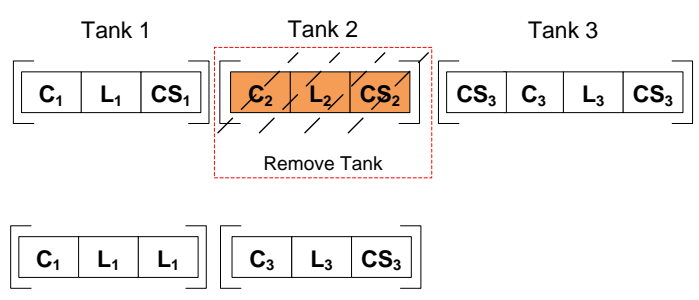

Figure 9: Structural removal of tank component within problem representation

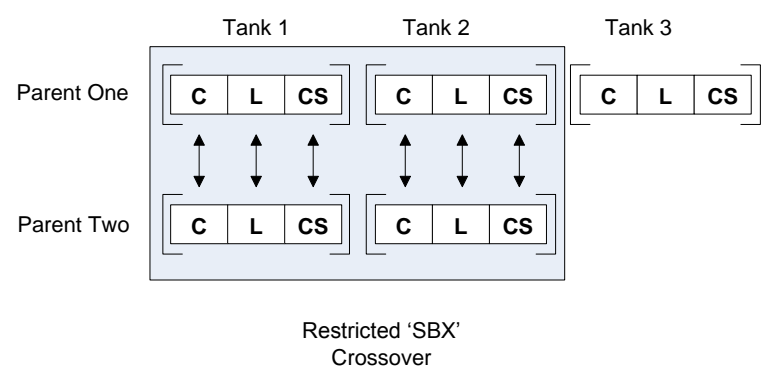

Figure 10: Restricted crossover within problem representation ter design problem the variable for tank number is excluded from SBX crossover, this is as a result of preliminary testing which found the tank number deviated to the mean value early in the design process, and this convergence disrupted the overall exploration for optimal solutions. The final node marker listed is also related to tank number, marked with Integer Discrete Mutation, this relates to the polynomial mutation module and how it handles discrete integer values. The polynomial operator of NSGAII and SPEA2 has been updated to include discrete values and where appropriate switch to simpler random discrete mutation which lies between the bounds of the chosen variable. In this instance tank number has bounds between 1 and 9 , and therefore mutation of the variable is simply a random choice within the bounds of the variable.

A final adaptation within NSGAII / SPEA2 and the SBX crossover module is how it handles structured and varied length representations. The representation used for this particular problem is structural in the sense it contains identifiable units that represent the LCR tanks used by the electrical equivalent circuit model, and varied, as the number of tanks can change depending on a controlling variable tank number. In order to perform successful crossover over two solutions with different lengths, as a result of tank number, a restriction is placed on the SBX crossover operation. The solution with the lowest number of structural units, in this case tanks, is chosen as the bounds of crossover, and therefore as shown in figure 10 only those tank units within both solutions have the crossover operation performed. This restriction stops the operator from performing crossover on the specific LCR tanks and associated variables between two parents where for one parent it does not exist as shown in figure 10 . This ensures structural integrity of the tree-based representation but as a result there is lower adaptation and exploration due to loss in variation of these 'end' LCR tanks.

The default algorithm parameters and experimental setup for both NSGAII and SPEA2 of the single and multi-level strategies are shown in table 5. The algorithmic parameters for mutation, crossover, distribution index and $\mathrm{k}$ strength are standard values taken from the literature. The population sizes were chosen to allow for a reasonable size spread of Pareto solutions and allow for both exploitation and exploration of the search space within a budget of 10,000 functional evaluations. 
The design process employed for the multi-level optimisation has three separate and isolated evolutionary algorithms that are structured to evolve their own population of solutions over a fixed period of generations or functional evaluations. Each of the separate levels employs the same default algorithmic parameters outlined in table 5, while the representation remains unchanged. The default multi-level evaluation template is shown in figure 11.

In order to transfer solutions from one level to another a migrator module is required. The migrator module is designed to handle the transfer of individual or sets of solutions from one population set to another. The migrator module contains a migration percentage parameter to indicate the number of individuals to transfer from one population set to another. The selection of individuals is based upon the same process within the standard NSGAII or SPEA2 algorithms, for example Pareto ranking and crowding, selecting the chosen percentage of individuals similar to truncation. If necessary as is the case here the chosen solutions are re-evaluated at the level specific analysis and then given a level specific rank and crowding value. A combination of both population sets then occurs similar to the standard Pareto ranking and crowding replacement to give the final population set. Successful migrants therefore remain within the final population while those of worse rank do not. Four migrator modules are utilized in this particular design strategy to allow individuals to move between neighbouring levels. The values for migration percentage along with the cycle count when migration is invoked are shown in table 6 .

\section{Case Studies}

In order to effectively test the robustness of this design methodology for MEMS bandpass filter synthesis a range of frequencies are chosen. Three bandpass filter case studies are outlined in table 3 beginning with a relatively low frequency taken from $\left[{ }^{43}\right]$, two more bandpass filter problems are introduced at the $20 \mathrm{kHz}$ and $100 \mathrm{kHz}$ range.

The design parameters are the same as those listed in table 2 except in the case of capacitance and coupling spring capacitance. The central frequency of the bandpass filter is determined primarily by the frequencies of the resonators that make up the network, of which these are heavily influ- enced by the mass and stiffness of the device ${ }^{31}$. In order to allow synthesis of bandpass filter transmissions which lie within the vicinity of the target central frequency the bounds for capacitance have been varied so initial design lies within the region of interest at the start of the design process as shown in table 4. The use of a design of experiments (DoE) is a common approach to choosing the bounds of design variables when applying evolutionary optimization $\left[{ }^{46}\right]\left[{ }^{47}\right]$.

The resistance component of the LCR tanks within the electrical equivalent circuit models is calculated using equation 4 which is linked to the tanks inductance and capacitance values as seen in equations 5 and 6 and follows similar approaches outlined in the literature [33]. The quality factor or $\mathrm{Q}$ value is fixed rather than calculated to a value of 40,000 which is consistent with the fabricated polysilicon folded flexure resonator within a vacuum, and has been outlined in [33]. Each case study as outlined in table 3 is fixed to a specific range where points are sampled at specific frequencies and then used to evaluate the two objectives outlined previously as seen in figures 5 and 6 . These were a range of $[0 \mathrm{~Hz}-10 \mathrm{kHz}]$ for case study 1 resulting in 10,000 sampling points, and $[0 \mathrm{~Hz}-$ $25 \mathrm{kHz}]$ and $[85 \mathrm{kHz}-110 \mathrm{kHz}]$ for case studies 2 and 3 respectively, resulting in 25,000 sampling points. Preliminary testing had discovered that given the size of the stop band in relation to sampling size, frequency transmissions would predominantly converge to having incredibly low magnitudes in such as to avoid having a high error as a result of the stop band $-20 \mathrm{~dB}$ target limit, as shown in figure 5. As a result weighting factors for the sum of the stop bands were set to divide the value by 9 and 25 in order for the algorithm to not focus to heavily on optimising the stop band. After synthesis of the electrical equivalent bandpass filter circuit models the values can then in the future be converted into their mechanical equivalent values using the equations of $4-8$ and then used as objectives for device level design optimisation of a folded flexure resonator. The multi-level strategy outlined previously looks to decompose the optimization process over a number of hierarchical levels, each with there own set of evaluation parameters. In a standard EA each functional evaluation is an outcome of any newly evolved solution and with constraint information provides information on the quality of the new design. The more functional evaluations that 


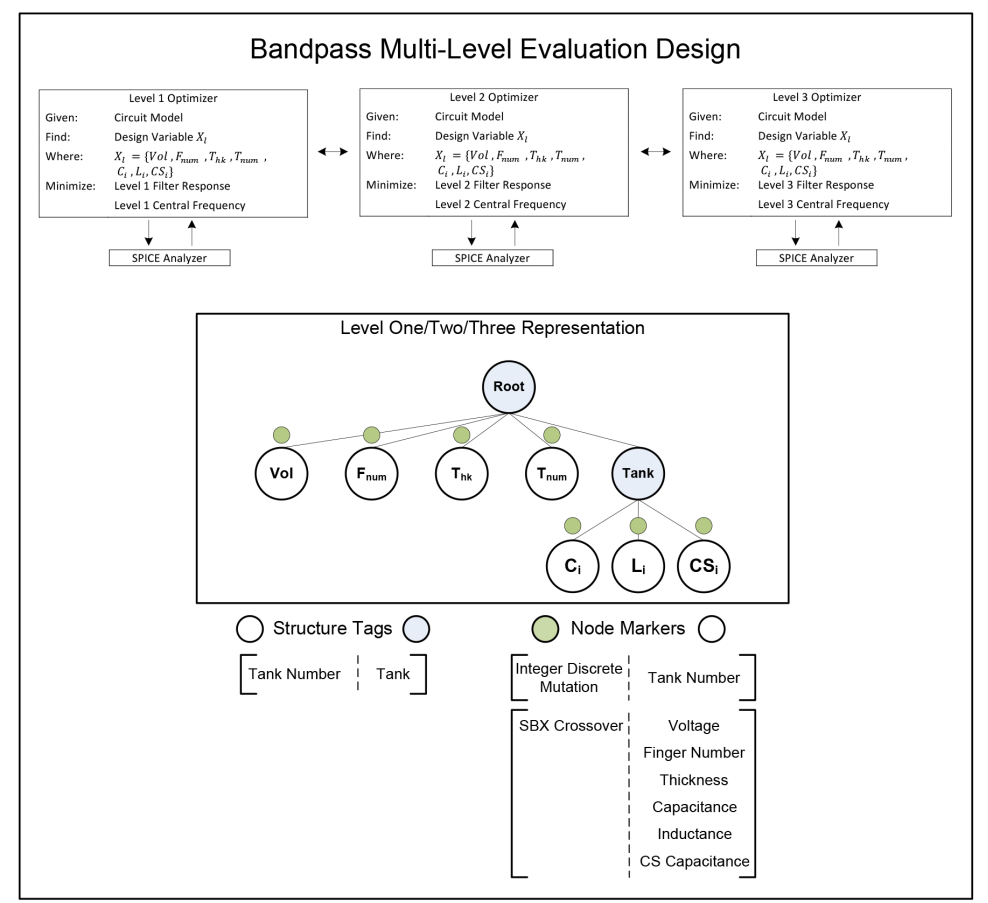

Figure 11: Bandpass multi-level evaluation design template, with overview of problem, default representation, associated structure tags and node markers

Table 3: Case Study Parameter Ranges

\begin{tabular}{cccc}
\hline & Case Study One & Case Study Two & Case Study Three \\
\hline Passband & $312 \mathrm{~Hz} 1000 \mathrm{~Hz}$ & $19.5 \mathrm{kHz} \quad 20.5 \mathrm{kHz}$ & $99.5 \mathrm{kHz} \quad 100.5 \mathrm{kHz}$ \\
Stopband 1 & $1 \mathrm{~Hz} 312 \mathrm{~Hz}$ & $1 \mathrm{~Hz} 19.5 \mathrm{kHz}$ & $85 \mathrm{kHz} 99.5 \mathrm{kHZ}$ \\
Stopband 2 & $1000 \mathrm{~Hz} 10 \mathrm{kHz}$ & $20.5 \mathrm{kHz} 25 \mathrm{kHz}$ & $100.5 \mathrm{kHz} 110 \mathrm{kHz}$ \\
Central Frequency & $656 \mathrm{~Hz}$ & $20 \mathrm{kHz}$ & $100 \mathrm{kHz}$ \\
\hline
\end{tabular}

Table 4: Circuit Design Variable Parameters

\begin{tabular}{cllllll}
\hline & \multicolumn{2}{l}{ Case Study } & One & \multicolumn{2}{c}{ Case Study Two } & \multicolumn{2}{c}{ Case Study Three } \\
\hline & Lower & Upper & Lower & Upper & Lower & Upper \\
& Values & Values & Values & Values & Values & Values \\
\hline Vesiable Type & - & - & - & - & - & - \\
& $1 \mathrm{e}-15$ & $1 \mathrm{e}-11$ & $1 \mathrm{e}-17$ & $1 \mathrm{e}-14$ & $1 \mathrm{e}-18$ & $1 \mathrm{e}-15$ \\
Capacitance $(\mathrm{F})$ & $\mathrm{F})$ & $1 \mathrm{e}-11$ & $1 \mathrm{e}-17$ & $1 \mathrm{e}-14$ & $1 \mathrm{e}-18$ & $1 \mathrm{e}-15$ \\
\hline
\end{tabular}


Table 5: Algorithm parameters

\begin{tabular}{cc}
\hline Algorithm Parameter & Value \\
\hline Probability of SBX Crossover & 0.8 \\
Probability of Mutation & 0.05 \\
Distribution Index for crossover & 20 \\
Distribution Index for mutation & 20 \\
SPEA2 Strength 'K' & 1 \\
Population Size & 100 \\
Offspring Size & 100 \\
Selection Size & 100 \\
Total Functional Evaluations & 10,000 \\
Runs & 5 \\
\hline
\end{tabular}

Table 6: Migrator parameters for multi-level evaluation strategy

\begin{tabular}{llll}
$\begin{array}{l}\text { Migration } \\
\text { Level }\end{array}$ & $\begin{array}{l}\text { Destination } \\
\text { Level }\end{array}$ & $\begin{array}{l}\text { Migration } \\
\text { Percentage }\end{array}$ & $\begin{array}{l}\text { Cycle } \\
\text { Count }\end{array}$ \\
\hline Level 1 & Level 2 & 20 & 4 \\
Level 2 & Level 1 & 20 & 4 \\
Level 2 & Level 3 & 20 & 4 \\
Level 4 & Level 3 & 20 & 4 \\
\hline
\end{tabular}

can occur the better, as it allows for further search within the design space.

The multi-level evaluation algorithm employs three separate evaluation characteristics all within the same functional budget of the standard approach. Each of the three levels are visualised in figure 12 , here level 1 covers the same range as the original single level analysis but samples only a fraction of the original points, while level 2 employs a different strategy of a restricted frequency window with a focus on the bandpass region of interest using a similar sampling size. The final level cover the entire frequency range of for example $1 \mathrm{~Hz}$ to $15 \mathrm{kHz}$ and samples each of the frequency points.

Table 7 outlines three levels of evaluation within the circuit simulator SPICE for analysis of the bandpass circuit model. The two lower levels, levels 1 and 2 provide a basic level of analysis of the frequency transmission at half of the cost in comparison with the standard analysis employed in the single level strategy. This is reflected in a smaller sampling size. Level 3 provides full range and sampling size for analysis, mirroring what is shown in figure 12. The same number of functional evaluations is used throughout the multi-level strategy process.

\section{Results}

The results are divided along each of the case studies undertaken in this paper. For each set of results there are tables for hypervolume values containing the upper, mean and lower sets, with the nadir point contained in brackets below. The best results obtained from each run ranked by the filter objective are also presented. In addition the final Pareto sets and the filter response for the best solution found by each strategy and algorithm are presented.

Overall there is no clear winner in terms of performance between the two separate MOEAs, with both NSGAII and SPEA2 altering with the best solutions over the three case studies presented. However it is clear that for both algorithms it is possible to synthesis solutions which match our targeted bandpass characteristics, however with a diminishing return as you move from case study one to three, a result in the shift in frequency range. Moving to the hypervolume results across the three cases studies, the strategies employed show a similar disparity with that of the differing algorithms with the single level strategy performing better on some experimental results and multi-level strategy working better on others. Delving into the best solutions produced and there bandpass filter responses shows that the first case study proved the easiest of the three in terms of solving and optimizing a desired bandpass filter response. The ability to alter the number of MEMS filters in the solution representation proved to be beneficial to the overall process showing a strong link with increased numbers and better solutions. Across the two case studies that used the multi-level approach results point to improved or comparable designs over those found by the single level strategy, though once again there is a dropoff in quality in case study three for both. Finally the population sets presented across the three case studies highlight two conflicting patterns, one shown throughout most of case study one and two of a characteristic Pareto front, while the other is of a convergence to a single or similar solution in the search space as shown in case study 3 results. Considering that the spread of population solutions is often determined by the central frequency objective, those solutions which contain multiple peaks, often found in case studies one and two may allow for a larger spread of solutions in relation to those which only contain a single peak. 


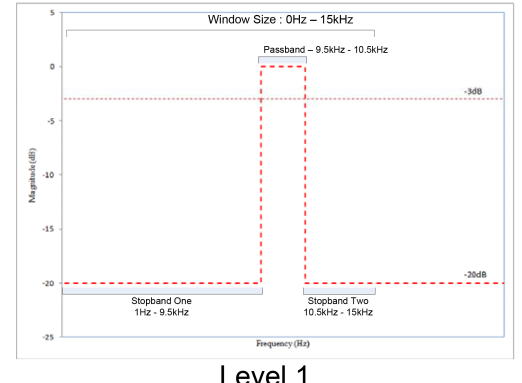

Level 1

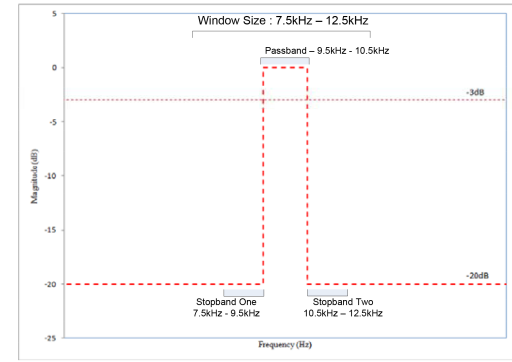

Level 2

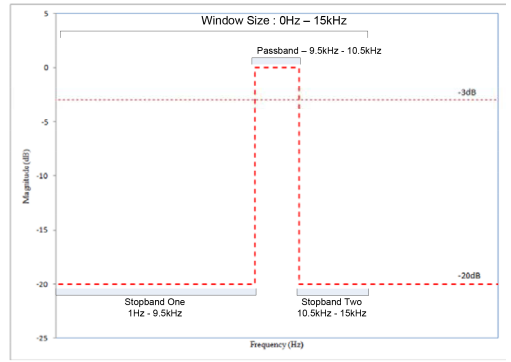

Level 3

Figure 12: Multi-level evaluation circuit model analysis sampling size and range characteristics - Sampling frequency ranges with full ranges $1 \mathrm{~Hz} 15 \mathrm{kHz}$ (level 1) and (level 3) and reduced range $7.5 \mathrm{kHz} 15 \mathrm{kHz}(\mathrm{Level}$ 2)

Table 7: Multi-Level Evaluation Circuit Model Analysis Parameters

\begin{tabular}{cccc}
\hline Case Study 2 & Level 1 & Level 2 & Level 3 \\
\hline Passband & $19.5 \mathrm{kHz} 20.5 \mathrm{kHz}$ & $19.5 \mathrm{kHz} 20.5 \mathrm{kHz}$ & $19.5 \mathrm{kHz} 20.5 \mathrm{kHz}$ \\
Stopband 1 & $1 \mathrm{~Hz} 19.5 \mathrm{kHz}$ & $13.75 \mathrm{kHz} 19.5 \mathrm{kHz}$ & $1 \mathrm{~Hz} 19.5 \mathrm{kHz}$ \\
Stopband 2 & $20.5 \mathrm{kHz} 25 \mathrm{kHz}$ & $20.5 \mathrm{kHz} 26.25 \mathrm{kHz}$ & $20.5 \mathrm{kHz} 25 \mathrm{kHz}$ \\
Sampling Size & 12500 & 12500 & 25000 \\
Central Frequency & $20 \mathrm{kHz}$ & $20 \mathrm{kHz}$ & $20 \mathrm{kHz}$ \\
\hline Case Study 3 & Level 1 & Level 2 & Level 3 \\
\hline Passband & $99.5 \mathrm{kHz} 100.5 \mathrm{kHz}$ & $99.5 \mathrm{kHz} 100.5 \mathrm{kHz}$ & $99.5 \mathrm{kHz} 100.5 \mathrm{kHz}$ \\
Stopband 1 & $85 \mathrm{kHz} 99.5 \mathrm{kHz}$ & $93.75 \mathrm{kHz} 99.5 \mathrm{kHz}$ & $85 \mathrm{kHz} 99.5 \mathrm{kHz}$ \\
Stopband 2 & $100.5 \mathrm{kHz} 110 \mathrm{kHz}$ & $100.5 \mathrm{kHz} 106.25 \mathrm{kHz}$ & $100.5 \mathrm{kHz} 110 \mathrm{kHz}$ \\
Sampling Size & 12500 & 12500 & 25000 \\
Central Frequency & $100 \mathrm{kHz}$ & $100 \mathrm{kHz}$ & $100 \mathrm{kHz}$ \\
\hline
\end{tabular}

Case Study 1 - NSGAII

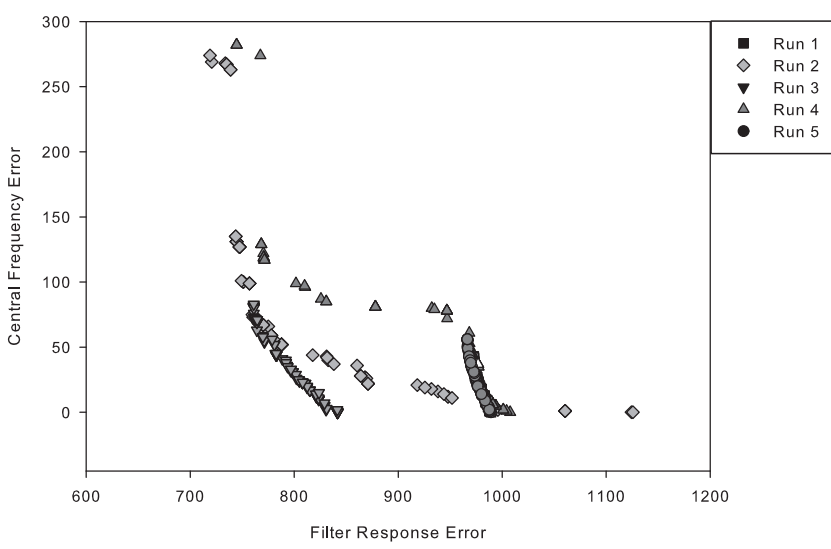

Case Study 1 - SPEA2

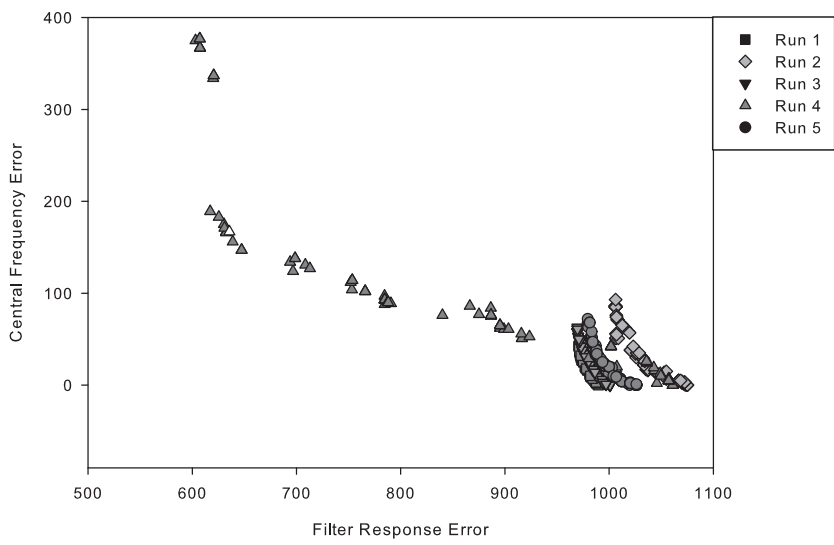

Figure 13: Case study 1 final population sets for NSGAII and SPEA2 
Case Study 2 - NSGAII
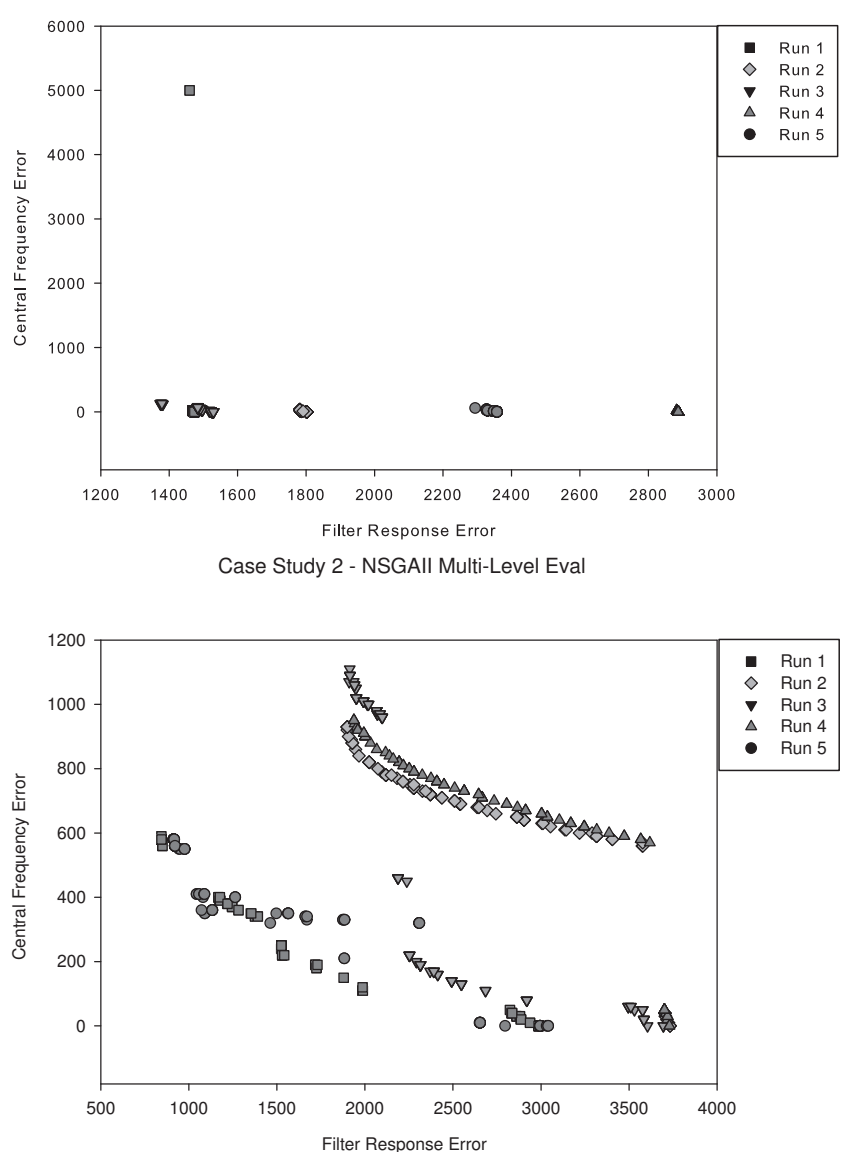

Case Study 2- SPEA2

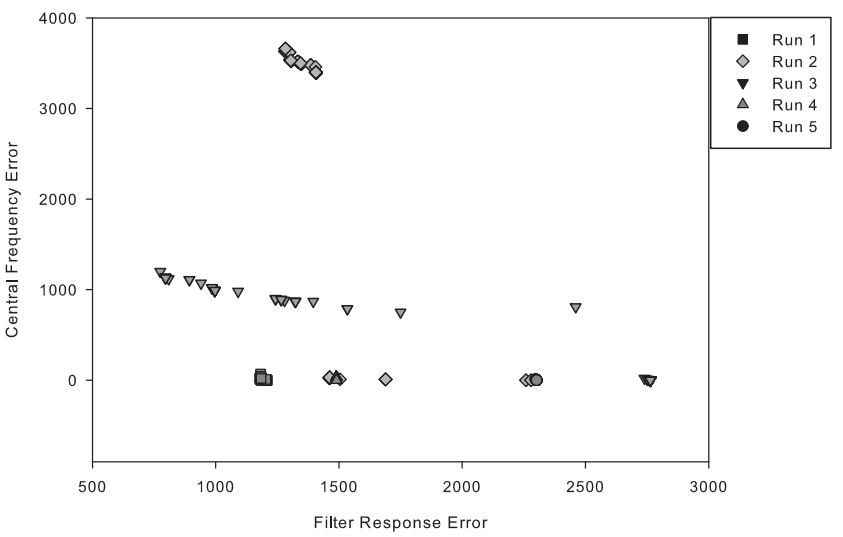

Case Study 2 - SPEA2 Multi-Level Eval

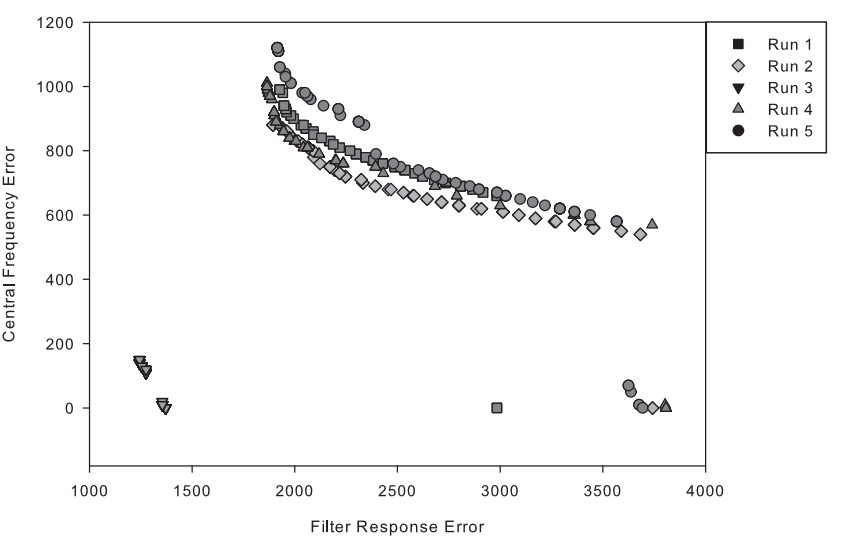

Figure 14: Case study 2 final population sets for both single and multi-level NSGAII and SPEA2 
Case Study 3 - NSGAII

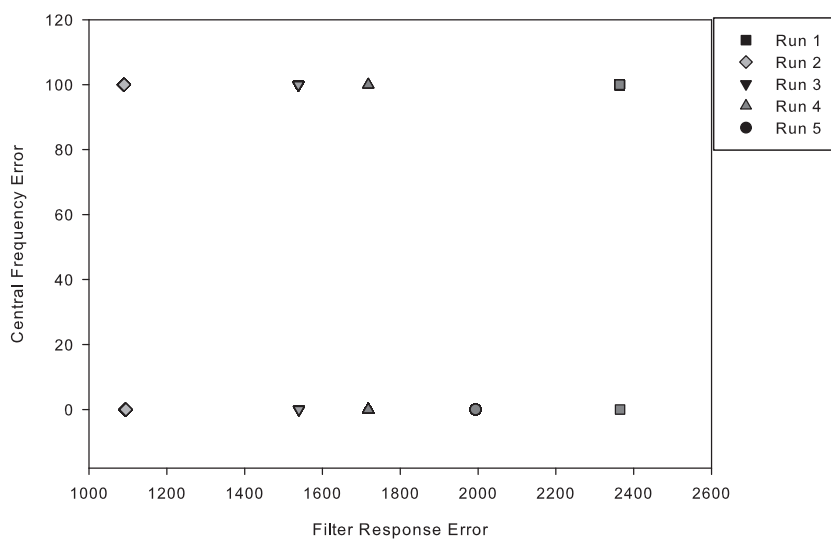

Case Study 3 - NSGAll Multi-Level Eval

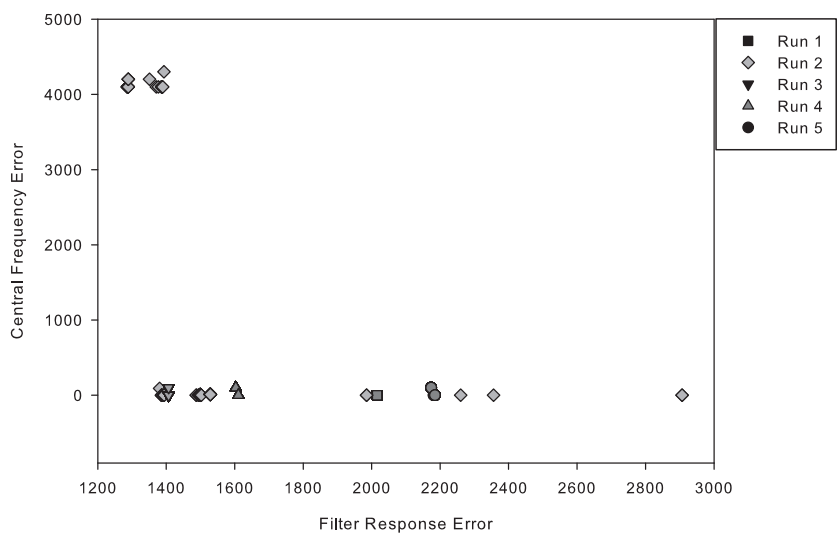

Case Study 3 - SPEA2

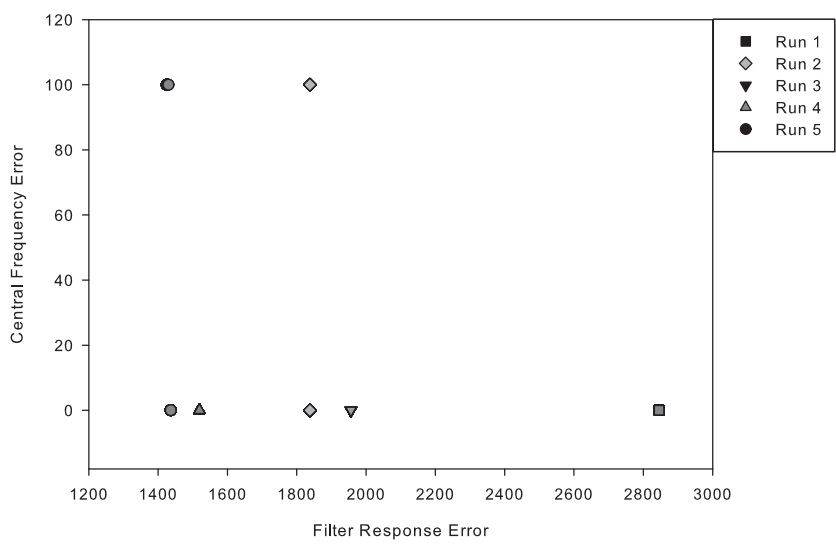

Case Study 3 - SPEA2 Multi-Level Eval

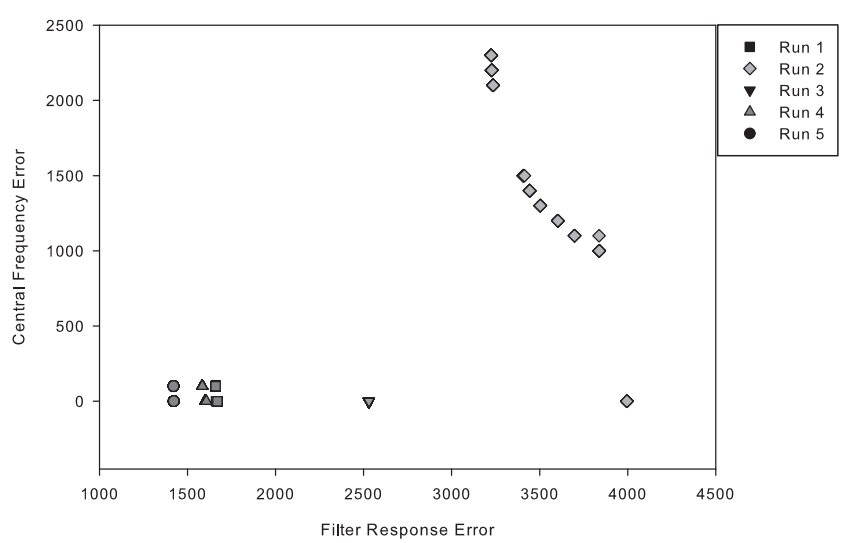

Figure 15: Case study 3 final population sets for both single and multi-level NSGAII and SPEA2

NSGAIl Case Study 1 - Filter Response

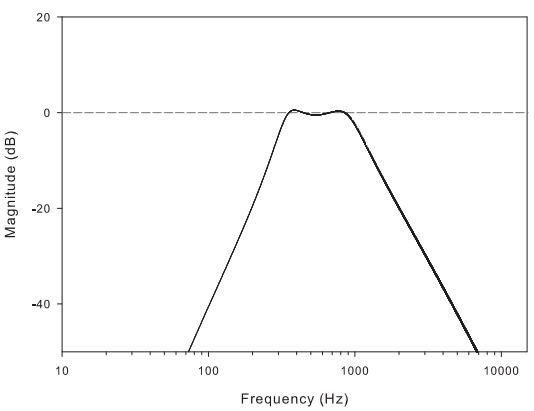

SPEA2 Case Study 1 - Filter Response

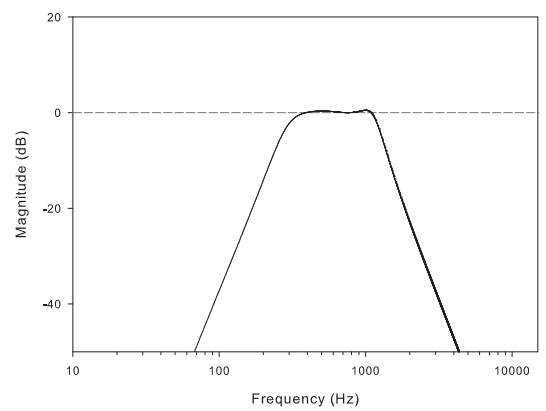

Figure 16: Case study 1 best filter responses for NSGAII and SPEA2 

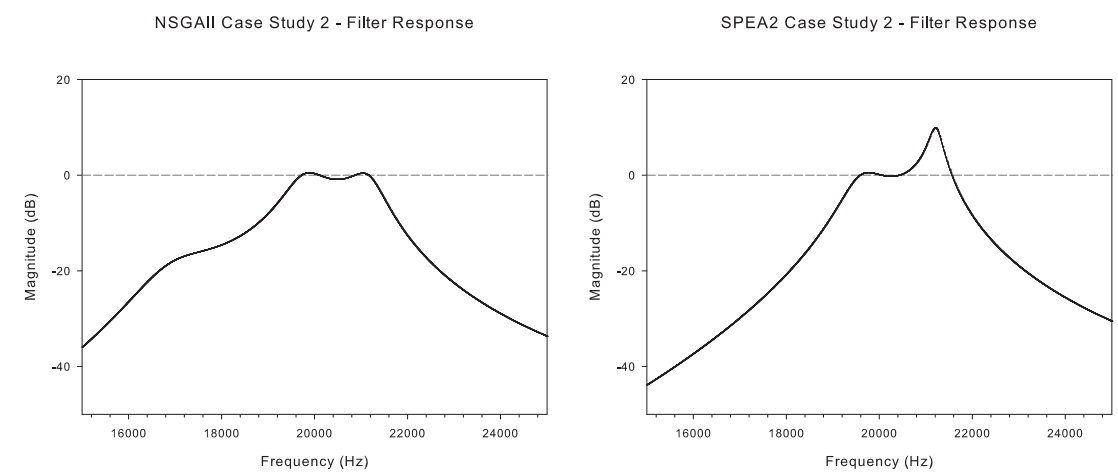

NSGAll Multi-Level Eval Case Study 2 - Filter Response

SPEA2 Multi-Level Eval Case Study 2 - Filter Response
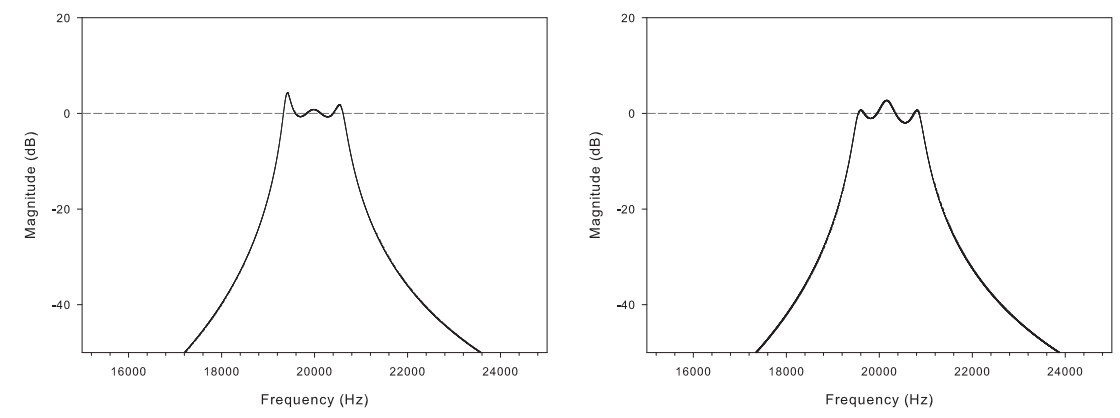

Figure 17: Case study 2 best filter responses for both single and multi-level NSGAII and SPEA2

NSGAll Case Study 3 - Filter Response

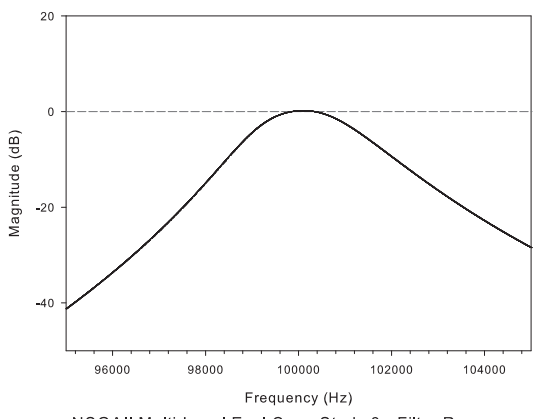

NSGAIl Multi-Level Eval Case Study 3 - Filter Response

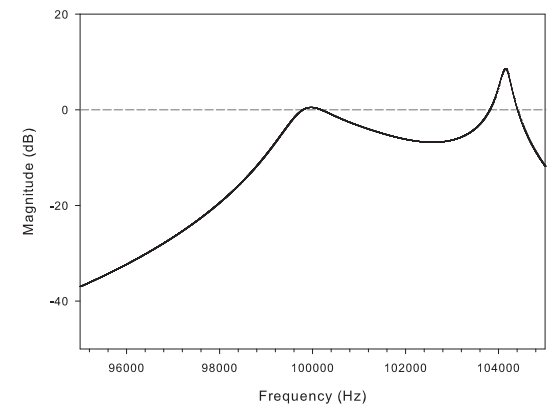

SPEA3 Case Study 3 - Filter Response

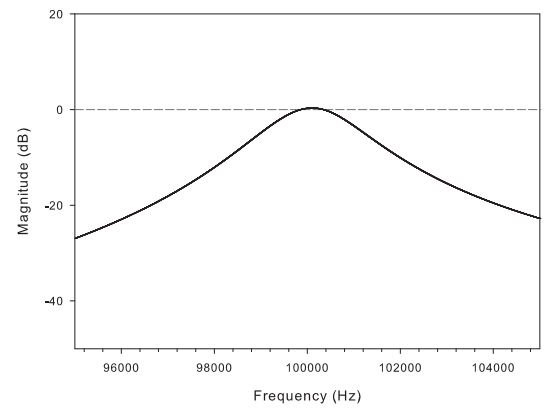

SPEA2 Multi-Level Eval Case Study 3 - Filter Response

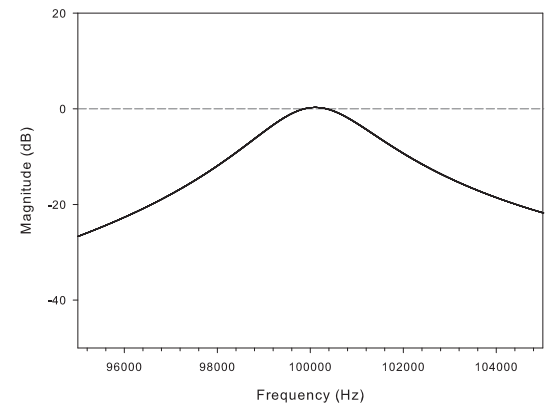

Figure 18: Case study 3 best filter responses for both single and multi-level NSGAII and SPEA2 
Table 8: Case Study 1 Hypervolume Results for Both NSGAII and SPEA2 algorithms

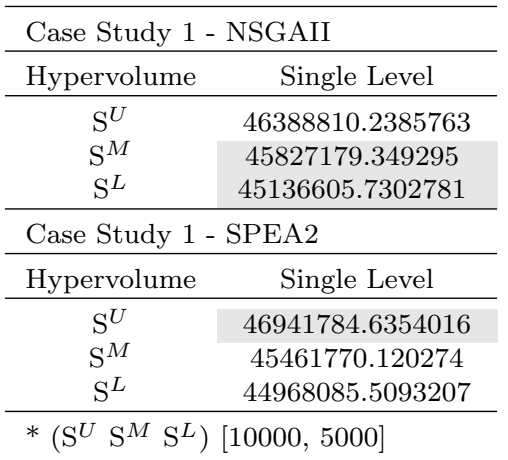

Table 9: Case Study 2 Hypervolume Results for Single and Multi-level Strategies for Both NSGAII and SPEA2 algorithms

\begin{tabular}{|c|c|c|}
\hline \multicolumn{3}{|c|}{ Case Study 2 - NSGAII } \\
\hline Hypervolume & Single Level & Multi-level Evaluation \\
\hline $\mathrm{S}^{U}$ & 43111940.7527106 & 45285426.4136971 \\
\hline $\mathrm{S}^{M}$ & 40194312.595487 & 41708785.5083203 \\
\hline $\mathrm{S}^{L}$ & 35584358.6733377 & 39279249.8424671 \\
\hline \multicolumn{3}{|c|}{ Case Study 2 - SPEA2 } \\
\hline Hypervolume & Single Level & Multi-level Evaluation \\
\hline $\mathrm{S}^{U}$ & 44467661.4434953 & 43765584.8163427 \\
\hline $\mathrm{S}^{M}$ & 42519320.426128 & 40222158.512744 \\
\hline $\mathrm{S}^{L}$ & 38512081.9945289 & 39136299.522289 \\
\hline
\end{tabular}

Table 10: Case Study 3 Hypervolume Results for Single and Multi-level Strategies for Both NSGAII and SPEA2 algorithms

\begin{tabular}{|c|c|c|}
\hline \multicolumn{3}{|c|}{ Case Study 3 - NSGAII } \\
\hline Hypervolume & Single Level & Multi-level Evaluation \\
\hline $\mathrm{S}^{U}$ & 44557081.7639095 & 43181322.8212017 \\
\hline $\mathrm{S}^{M}$ & 41297706.822393 & 41437538.134945 \\
\hline $\mathrm{S}^{L}$ & 38179128.8033502 & 39132899.9901634 \\
\hline \multicolumn{3}{|c|}{ Case Study 3 - SPEA2 } \\
\hline Hypervolume & Single Level & Multi-level Evaluation \\
\hline $\mathrm{S}^{U}$ & 42876300.5406364 & 42898111.7480578 \\
\hline $\mathrm{S}^{M}$ & 40417061.663394 & 39365529.280350 \\
\hline $\mathrm{S}^{L}$ & 35773232.7228355 & 32797218.5021526 \\
\hline
\end{tabular}

\section{Discussion}

Automation of the design process is an important tool for any designer. In the field of microelectromechanical systems designer are often faced with the need to use numerous modeling and simulation tools that span across multiple disciplines. Coupling methods of modeling and simulation with that of automated design synthesis and optimization is of great benefit. In addition often these tools are highly computationally expensive and can slow down the design process as a result.

Research into methods for overcoming this problem by looking to speed up the design process but without a loss to overall design optimization quality is also a desired goal. This work set out to investigate the role multi-objective evolutionary algorithms could play in the evolution and optimization of designs for MEMS bandpass filters. This involved the creation of a novel approach to the optimization of bandpass filters through an electrical equivalent circuit modeling method coined GAECM. In addition this was expanded to include the use of alternative strategies for overcoming computational expense and expanding the search process through the use of a multi-level evaluation strategy. In this demonstration the use of multiobjective evolutionary algorithms proved successful in evolving and optimizing designs for MEMS bandpass filters that match the targeted bandpass characteristics outlined. The restructuring of the optimization process into a multi-level evaluation strategy allowed for more solutions to be created, in comparison with the single level strategy. In some instances this only led to solutions converging to a stable Pareto front, in others the ability to break free. However in either case it provides the designer with more solutions to choose from at the end of the process. In addition the multi-level strategy opens up the design process to different and varying abstractions of the design search space as a result of lowering the number of points sampled and the distribution from where they were sampled over the three different levels. The GAECM approach outlined in this paper was able to provide comparable bandpass filter transmissions to those within the literature for the current state of the art in automated design $\left[{ }^{43}\right]$ as well as expand upon it through additional case study examples. In terms of overall performance the approach shows a significant reduction in functional evaluations, 10,000 compared with 2.6 million required to solve this design prob- 
Table 11: NSGAII Bandpass Filter Results

\begin{tabular}{|c|c|c|c|c|c|}
\hline \multicolumn{6}{|c|}{ Case Study 1 - NSGAII } \\
\hline Test & Index & Filter Objective & Central Frequency Objective & Voltage & Tank Number \\
\hline 1 & 2 & 972.623 & 43 & 110.15 & 2 \\
\hline 2 & 66 & 719.019 & 274 & 142.99 & 3 \\
\hline 3 & 25 & 761.092 & 82 & 13.73 & 3 \\
\hline 4 & 1 & 743.996 & 282 & 117.16 & 3 \\
\hline 5 & 0 & 966.501 & 56 & 101.75 & 2 \\
\hline \multicolumn{6}{|c|}{ Case Study 2 - NSGAII } \\
\hline Test & Index & Filter Objective & Central Frequency Objective & Voltage & Tank Number \\
\hline 1 & 0 & 1459.958 & 5000 & 121.49 & 2 \\
\hline 2 & 4 & 1781.875 & 30 & 168.33 & 2 \\
\hline 3 & 0 & 1374.729 & 120 & 83.02 & 3 \\
\hline 4 & 5 & 2882.119 & 20 & 10.72 & 2 \\
\hline 5 & 1 & 2293.985 & 60 & 26.73 & 2 \\
\hline \multicolumn{6}{|c|}{ Case Study 2 - NSGAII Multi-level Evaluation } \\
\hline Test & Index & Filter Objective & Central Frequency Objective & Voltage & Tank Number \\
\hline 1 & 3 & 844.337 & 580 & 121.97 & 3 \\
\hline 2 & 12 & 1900.580 & 930 & 123.99 & 2 \\
\hline 3 & 23 & 1910.717 & 1070 & 180.77 & 2 \\
\hline 4 & 0 & 1937.431 & 950 & 51.86 & 2 \\
\hline 5 & 0 & 912.560 & 580 & 146.91 & 3 \\
\hline \multicolumn{6}{|c|}{ Case Study 3 - NSGAII } \\
\hline Test & Index & Filter Objective & Central Frequency Objective & Voltage & Tank Number \\
\hline 1 & 0 & 2364.157 & 100 & 19.54 & 2 \\
\hline 2 & 0 & 1088.497 & 100 & 59.29 & 7 \\
\hline 3 & 1 & 1538.346 & 100 & 109.89 & 3 \\
\hline 4 & 1 & 1717.592 & 100 & 15.76 & 3 \\
\hline 5 & 0 & 1993.571 & 0 & 16.40 & 2 \\
\hline \multicolumn{6}{|c|}{ Case Study 3 - NSGAII Multi-level Evaluation } \\
\hline Test & Index & Filter Objective & Central Frequency Objective & Voltage & Tank Number \\
\hline 1 & 0 & 2015.822 & 0 & 11.19 & 2 \\
\hline 2 & 0 & 1285.824 & 4100 & 125.9 & 3 \\
\hline 3 & 0 & 1406.135 & 100 & 30.61 & 3 \\
\hline 4 & 0 & 1603.193 & 100 & 142.06 & 3 \\
\hline 5 & 0 & 2173.267 & 100 & 117.77 & 3 \\
\hline
\end{tabular}


Table 12: SPEA2 Bandpass Filter Results

\begin{tabular}{|c|c|c|c|c|c|}
\hline \multicolumn{6}{|c|}{ Case Study 1 - SPEA2 } \\
\hline Test & Index & Filter Objective & Central Frequency Objective & Voltage & Tank Number \\
\hline 1 & 10 & 970.971 & 43 & 104.16 & 3 \\
\hline 2 & 1 & 1006.062 & 86 & 102.15 & 2 \\
\hline 3 & 82 & 969.495 & 64 & 12.03 & 2 \\
\hline 4 & 39 & 601.056 & 334 & 17.74 & 3 \\
\hline 5 & 30 & 978.562 & 66 & 139.57 & 2 \\
\hline \multicolumn{6}{|c|}{ Case Study 2 - SPEA2 } \\
\hline Test & Index & Filter Objective & Central Frequency Objective & Voltage & Tank Number \\
\hline 1 & 0 & 1177.699 & 0 & 96.159 & 3 \\
\hline 2 & 12 & 1280.012 & 3630 & 103.35 & 2 \\
\hline 3 & 6 & 775.304 & 1200 & 31.89 & 3 \\
\hline 4 & 3 & 1489.349 & 30 & 119.68 & 2 \\
\hline 5 & 0 & 2297.576 & 10 & 11.13 & 2 \\
\hline \multicolumn{6}{|c|}{ Case Study 2 - SPEA2 Multi-level Evaluation } \\
\hline Test & Index & Filter Objective & Central Frequency Objective & Voltage & Tank Number \\
\hline 1 & 43 & 1926.593 & 990 & 125.06 & 2 \\
\hline 2 & 16 & 1894.081 & 880 & 147.47 & 2 \\
\hline 3 & 10 & 1244.274 & 150 & 62.34 & 3 \\
\hline 4 & 5 & 1861.903 & 1010 & 19.52 & 2 \\
\hline 5 & 40 & 1914.683 & 1120 & 167.87 & 2 \\
\hline \multicolumn{6}{|c|}{ Case Study 3 - SPEA2 } \\
\hline Test & Index & Filter Objective & Central Frequency Objective & Voltage & Tank Number \\
\hline 1 & 0 & 2845.353 & 0 & 193.06 & 2 \\
\hline 2 & 0 & 1837.836 & 0 & 106.05 & 3 \\
\hline 3 & 0 & 1956.387 & 0 & 15.24 & 2 \\
\hline 4 & 0 & 1518.380 & 0 & 19.36 & 3 \\
\hline 5 & 0 & 1424.506 & 100 & 101.95 & 3 \\
\hline \multicolumn{6}{|c|}{ Case Study 3 - SPEA2 Multi-level Evaluation } \\
\hline Test & Index & Filter Objective & Central Frequency Objective & Voltage & Tank Number \\
\hline 1 & 1 & 1659.214 & 100 & 11.97 & 3 \\
\hline 2 & 0 & 3225.047 & 2300 & 152.84 & 2 \\
\hline 3 & 0 & 2530.561 & 0 & 186.43 & 2 \\
\hline 4 & 0 & 1583.347 & 100 & 29.79 & 3 \\
\hline 5 & 0 & 1420.351 & 100 & 125.09 & 3 \\
\hline
\end{tabular}


Case Study 1 - Best Random Filter Response

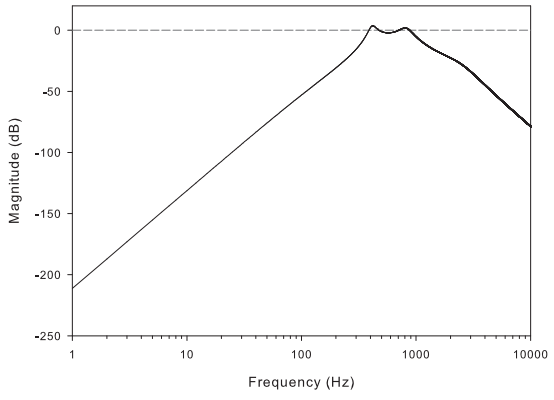

Case Study 2 - Best Random Filter Response
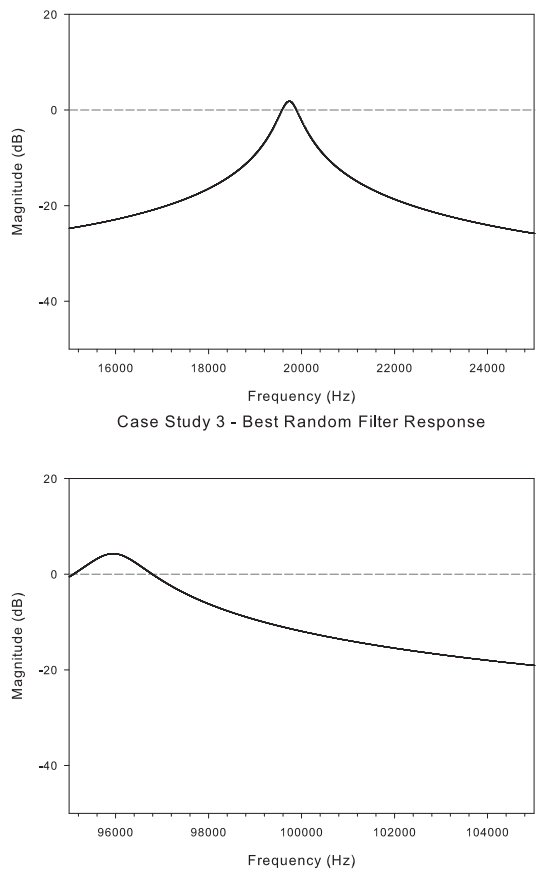

Case Study 1 - Central Frequency Histogram

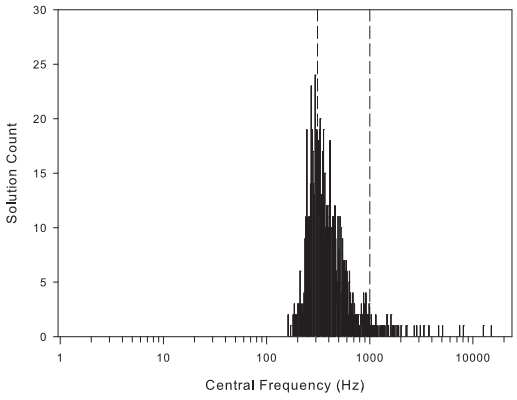

Case Study 2 - Central Frequency Histogram

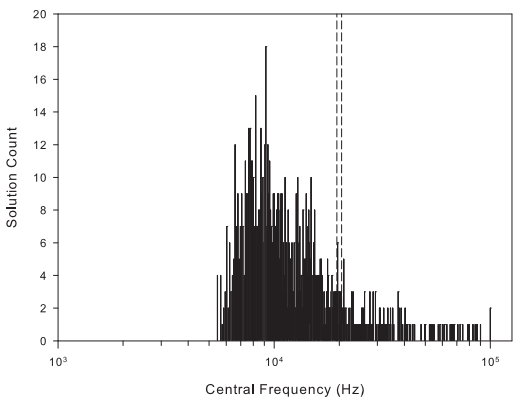

Case Study 3 - Central Frequency Histogram

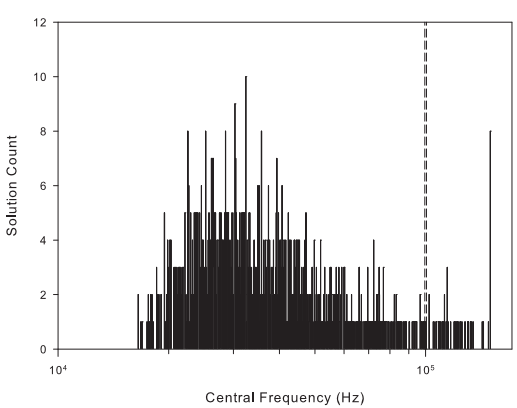

Figure 19: Best random filter response and central frequency histogram for band pass filter case studies, dashed lines mark band regions for each filter 
lem when compared with the state of the art $\left[{ }^{43}\right]$. However it is hard to make a direct comparison as both approaches have their benefits and drawbacks, in particular the GPBG method outlined in [ $\left.{ }^{43}\right]$ utilizes bond graphs which are open to more creative design then a rigid LCR circuit model. The electrical equivalent circuit model itself is also a simplified representation, with for example drawbacks in modelling as a one-port impedance device rather than two-port $\left[{ }^{31}\right]$, and representing the coupling shunt capacitors as massless ideal springs when in reality there is some finite mass which may affect filter transmission $\left.{ }^{32}\right]$. The use of a flexible representation has also proved to be useful, as highlighted in tables 11 and 12, where the best solutions have the higher number of LCR tanks in their solution designs, in some instances going to as high as 7 resonator tanks in case study 3 for NSGAII. There is however depreciation in quality as the frequency range is increased over the case studies. One explanation for this can be assigned to the initial start of the design process, and where the optimisation starts within the design search space. The chosen upper and lower bounds for the inductance and capacitance plays an important role in where the central frequency response of the filter lies within the frequency range. This is highlighted in figure 19, where for each case study, 10,000 randomly initialised solutions are created and analysed, and a histogram of their central frequency is plotted showing the individual count and where they lie for a given frequency. Beside these plots the best solution ranked by filter frequency from the 10,000 random solutions is shown. The $656 \mathrm{~Hz}$ bandpass filter of case study one highlights how successfully chosen upper and lower bounds can give rise to randomly initialised solutions that lie within the passband region of interest and provide solutions with good bandpass frequency transmissions. As the range of interest changes to $20 \mathrm{kHz}$ and then $100 \mathrm{kHz}$ it can be seen that for the bounds chosen for the design variables the solutions produced lie further away from the target passband ranges. The quality of the solutions is also lower with limited bandpass transmission shape, with case study two lying close to the target central frequency but consisting of a single resonator, while for case study three the best random solution lies even further away from the target central frequency. The effect of choosing the correct variable bounds at the start of the optimization process is clear and it is recommended that a design of experiments be used to identify the best boundary ranges for each variable. Care must also be given when choosing the level of sampling across a given frequency range for the lower level abstractions. The number of points affects how the bandpass filter shape is evaluated, and as a result the genotype to phenotype mapping will vary. This may be detrimental as it means it is harder to differentiate solutions if the sampling rate leads to a granular bandpass filter shape.

Overall however the use of electrical equivalent to mechanical equivalent conversion methods has proved successful and it provides a direct link between the system and device level optimisation through physical mechanical targets for device layout optimisation leading to a realization of the functional circuit models.

\section{Conclusions and Future Work}

Microelectromechnical systems design presents many challenges. The multidisciplinary nature of modeling, simulation and application of the devices and the computational expense and conflicting objectives are just some that face designers. To aid designers an automated single level multi-objective evolutionary algorithm approach has been developed for the design and optimization of MEMS bandpass filters. This is coupled with a new approach for MEMS bandpass modeling and optimization through an electrical equivalent modeling approach coined GAECM. In addition a multi-level evaluation strategy that uses hierarchical simulation to aid optimization was outlined and tested over a number of bandpass filter case studies. The new GAECM approach proved successful in evolving designs that gave comparable results to earlier work $\left[{ }^{43}\right]$, but at a fraction of the cost, needing only 10,000 functional evaluations in comparison to 2.6 million with the previous work. Also our designs were restricted to bounds that gave rise to feasible and realizable physical targets unlike previous attempts, by using the required electrical equivalent to mechanical equivalent conversion method presented in [ $\left.{ }^{33}\right]$. This allowed for the creation of filter designs that could be feasible and realizable in terms of fabrication of the resulting 2D layout designs. Future work will look to expand the multilevel evaluation strategy into other disciplines, in particular the device and physical levels of modeling and simulation. Here the computational cost of 
simulation is larger and therefore the benefits obtained will be greater as a result.

\section{Acknowledgments}

This work was funded by the EPSRC EXHUME project, grant number EP/K026348/1, through the kind support of the manufacturing informatics centre at Cranfield University.

\section{References}

[1] Hsu, T. R., (2008) MEMS and Microsystems Second Edition. Wiley, ISBN: 978-0-47008301-7

[2] Neeves, K. B., and Diamond, S., (2008) A membrane-based microfluidic device for controlling the flux of platelet agonists into flowing blood Lab Chip. 8(5). pp 701-709.

[3] Bechtold, T., Rudnyi, E. B. and Korvink, J. G. (2003) "Automatic Generation of Compact Electro-thermal Models for Semiconductor Devices", IEICE Transactions on Electronics, vol. 86, pp.459-65

[4] Hostis, F. l., Green, N. G., Morgan, H., and Akaisi, M., (2006) Solid state AC Electroosmosis Micro Pump on a Chip International Conference on Nanoscience and Nanotechnology, ICONN, Brisbane, Qld. pp 282-285. July, 2006.

[5] Ji, C-H., Yee, Y., Choi, J., Kim, S-h., and Bu, J-U., (2004) Electromagnetic 2 x 2 MEMS Optical Switch IEEE Journal of Selected Topics in Quantum Electronics, vol 10, No 3. pp 545550 .

[6] Ongkodjojo, A., and Tay, F. E. H., (2002) Global Optimization and Design for Microelectromechanical Systems Devices Based on Simulated Annealing Journal of Micromechanics and Microengineering, pp 878-897.

[7] Fedder, G. K., and Mukherjee, T., (1996) "Physical Design For Surface-Micromachined MEMS" In Proceedings of the 5th ACM/SIGDA Physical Design Workshop, Reston, VA USA, pp 53-60, April 15-17, 1996.

[8] Farnsworth, M.J., Tiwari, A., and Dorey, R., (2014) "Modelling, Simulation and Optimisation of a Piezoelectric Energy Harvester" Procedia CIRP, 22, pp 142-147

[9] Brenner, M.P., Lang, J.H., Li, J., Qiu, J. and Slocum, A. H, (2002) Optimum Design of MEMS Switch, Modeling and Simulation of Microsystems

[10] Han J.S. and Kwak, B. M, (2001) Robust optimal design of a vibratory microgyroscope considering fabrication errors. Journal of Micromechanics and Microengineering. 11, pp.662-71

[11] Zhou N, Agogino AM, Pister KS (2002) Automated Design Synthesis for Micro-ElectroMechanical Systems (MEMS). In: Proceedings of the ASME design automation conference, ASME CD ROM, Sept. 29Oct. 2 2002, Montreal, Canada

[12] Zhou, N., Clark, J.V., and Pister, K.S.J., (1998) Nodal Analysis for MEMS Design Using Sugar v0.5, Technical Proceedings of the 1998 International Conference on Modelling and Simulation of Microsystems, pp.308-313

[13] Campbell ML (2000) The A-Design Invention Machine: A Means of Automating and Investigating Conceptual Design. Ph.D Thesis. Carnegie Mellon University, Carnegie Institute of Technology, United States of America

[14] Ye W, Mukherjee S, MacDonald NC (1998) Optimal shape design of an electrostatic Comb Drive in microelectromechanical systems. J Microelectromechanical Syst 7:1626

[15] Fan Z, Wang J, Goodman ED (2005) An evolutionary approach for robust layout synthesis of MEMS. In: Proceedings of 2005 IEEE/ASME international conference on advanced intelligent mechatronics, pp 11861191

[16] Zhang Y (2006) MEMS design synthesis based on hybrid evolutionary computation, $\mathrm{PhD}$ thesis, Department of Civil and Environmental Engineering, University of California at Berkeley, USA

[17] Kamalian R (2004) Evolutionary synthesis of MEMS devices PhD thesis, University of California at Berkeley, California, United States of America.

[18] Cobb C, Zhang Y, Agogino AM (2006) An integrated MEMS design synthesis architecture using case-based reasoning and multi- objective genetic algorithms. In: Proceedings of 2006 SPIE smart materials, nano- and microsmart systems, SPIE, vol. 6414, No. 641419, ISBN: 9780819465221, Invited paper

[19] Farnsworth, M., Benkhelifa, E., Tiwari, A., Zhu, M., and Moniri, M., (2011) "An efficient evolutionary multi-objective framework 
for MEMS design optimisation: validation, comparison and analysis" Journal of Memetic Computing, Vol 3, 3, pp 175-197

[20] Karakasis, M., and Giannakoglou, K., (2003) Inexact information aided, Low-cost, distributed genetic algorithms for aerodynamic shape optimisation International Journal for Numerical Methods in Fliuds, Vol 43, (10-11), pp 1149-1166.

[21] Kampolis, I., and Giannakoglou, K., (2008) A Multilevel approach to single- and multiobjective aerodynamic optimization Computer Methods in Applied Mechanics and Engineering, 197. (33-40), pp 2963-2975

[22] Kampolis, I.C., and Giannakoglou, K. C. (2010) Synergetic use of different evaluation, parameterization and search tools within a multilevel optimization platform Applied Soft Computing, doi: 10.1016

[23] Liakopoulos, P. I. K., Kampolis, I. C., and Giannakoglou, K. C. (2008) Grid enabled, hierarchical distributed metamodel-assisted evolutionary algorithms for aerodynamic shape optimization Future Generation Computer Systems, Vol 24, pp701-708

[24] Kampolis, I. C., and Giannakoglou K. C. (2009) Distributed evolutionary algorithms with hierarchical evaluation Engineering Optimization, Vol 41, No 11, pp1037-1049

[25] Farnsworth, M., (2012) "Multi-level and multidisciplinary optimisation of microelectromechanical systems" PhD Thesis, Cranfield University, United Kingdom

[26] Farnsworth, M., Benkhelifa, E., Tiwari, A., Zhu, M., (2010) "A Multi-level Evolutionary Design Optimisation of MEMS: Methodology and Application" In Proceedings Third International Conference on Multidisciplinary Design Optimization and Applications

[27] Farnsworth M, Benkhelifa E, Tiwari A, Zhu M (2010) "A novel approach to multi-level evolutionary design optimization of a MEMS device". Evolvable systems: from biology to hardware, LNCS 6274:322334

[28] Senturia, S. D., (2001) Microsystem Design, Kluwer Academic Publishers. ISBN-0-79237246-8.

[29] Nguyen, C, T-C., Katchi, L. P. B., and Rebeiz, G. M., (1998) Micromachined Devices for Wireless Communications Proceedings IEEE, vol 86, no 8, pp. 1756-1768, Aug
[30] Mestrom, R. M. C., (2007) Microelectromechanical Oscillators a literature survey DCT Internal report, Eindhoven University of Technology,

[31] Bannon. F. D., Clark, J. R., and Nguyen, C. TC., (2000) High-Q HF Microelectromechanical Filters IEEE Journal of Solid-State Circuits, Vol 35. No 4. pp 512-526

[32] Jing, Q., Mukherjee, T., and Fedder, G., (1999) A Design Methodology for Micromechanical Bandpass Filters In Proceedings of 1999 IEEE/ACM/VIUF International Workshop on Behavioral Modeling and Simulation (BMAS 99), Oct 4-6, Orlando, Fl, USA

[33] Wang, K., and Nguyen, C. T-C. (1999) HighOrder Medium Frequency Micromechanical Electronic Filters Journal of MicroElectroMechanical Systems. Vol 8. No 4. pp 534-556

[34] Sykes, R. A., Smith, W. L., and Spencer, W. J., (1967) Monolithic crystal filters in IEEE International Conv. Rec. pt. II, March. pp 7893

[35] Rennick, R. C., (1973) An equivalent circuit approach to the design and analysis monolithic crystal filters IEEE Transactions. Sonic. Ultrason., vol SU-20, pp 347-354

[36] Krishnaswamy, S. V., Rosenbaum, J., Horwitz, S., Yale, C., and Moore, R. A., (1991) Compact FBAR Filters offer low-loss performance Microwaves and RF, pp. 127-136, Sept

[37] Lin, L., Nguyen, C. T-C., Howe, R. T., and Pisano, A. P., (1992) Micro Electromechanical Filters for Signal Processing Micro Electro Mechanical Systems

[38] Nguyen, C. T-C., Micromechanical Resonators for Oscillators and Filters Proceedings of the 1995 IEEE International Ultrasonics Symposium, Seattle, WA, pp 489-499, Nov-7-10. (1995)

[39] Tilmans, H. A. C., (1996) Equivalent circuit representation of electromechanical transducers: I. Lumped-parameter systems Journal Micromechanical Micro engineering. Vol 6, pp 157-176

[40] Deb, K., Agrawal, S., Pratap, A., and Meyarivan, T., (2000) A fast elitist non-dominated sorting genetic algorithm for multi-objective optimization: NSGA-II". In Proceedings of the 6-th International Conference Parallel Problem Solving from Nature (PPSN-VI), pp 849-858. 
[41] Zitzler, E., Laumanns, M., and Thiele, L., (2001) SPEA2: Improving the Strength Pareto Evolutionary Algorithm for Multiobjective Optimization Evolutionary Methods for Design Optimization and Control with Applications to Industrial Problems, Athens, Greece: International Center for Numerical Methods in Engineering, pp 95-100

[42] Nagel, L. W, and Pederson, D. O., (1973) SPICE (Simulation Program with Integrated Circuit Emphasis), Memorandum No. ERLM382, University of California, Berkeley, Apr.

[43] Fan, Z., Wang, J., Achiche, S., Goodman E., and Rosenberg R. (2008) Structured Synthesis of MEMS using evolutionary approaches Applied Soft Computing 8, pp 579-589

[44] Vandemeer, J., Kranz, M. and Fedder, G, (1997) Nodal Simulation of Suspended MEMS Multiple Degrees of Freedom, MEMS ASME: DSC-Vol. 62, pp.113-118.

[45] Vandemeer, J., Kranz, M. and Fedder, G, (1998) Hierarchical Representation and Simulation of Micromachined Inertial Sensors, Modeling and Simulation of Microsystems, Santa Clara, CA, April

[46] Reeves, C. R., and Wright, C., (1999) 'Genetic Algorithms and the Design of Experiments', Evolutionary Algorithms, Vol 111 of the series The IMA Volumes in Mathematics and its Applications pp 207-226

[47] Valipour, M., (2016) Optimization of neural networks for precipitation analysis in a humid region to detect drought and wet year alarms Meteorological Applications, Vol 23: pp 91100 
2016-10-11

\section{Multi-level and multi-objective design optimisation of a MEMS bandpass filter}

Farnsworth, Michael

Elsevier

Farnsworth M, Tiwari A, Zhu M, Multi-level and multi-objective design optimisation of a MEMS bandpass filter, Applied Soft Computing, Vol. 52, March 2017, pp. 642-656

http://dx.doi.org/10.1016/j.asoc.2016.10.007

Downloaded from Cranfield Library Services E-Repository 